\title{
Universality in the shape dependence of holographic Rényi entropy for general higher derivative gravity
}

\author{
Chong-Sun Chu ${ }^{a, b}$ and Rong-Xin Miao ${ }^{b}$ \\ ${ }^{a}$ Physics Division, National Center for Theoretical Sciences, National Tsing-Hua University, \\ 101 Section 2 Kuang Fu Road, Hsinchu 30013, Taiwan \\ ${ }^{b}$ Department of Physics, National Tsing-Hua University, \\ 101 Section 2 Kuang Fu Road, Hsinchu 30013, Taiwan
}

E-mail: cschu@phys.nthu.edu.tw, miaorongxin.physics@gmail.com

ABSTRACT: We consider higher derivative gravity and obtain universal relations for the shape coefficients $\left(f_{a}, f_{b}, f_{c}\right)$ of the shape dependent universal part of the Rényi entropy for four dimensional CFTs in terms of the parameters $\left(c, t_{2}, t_{4}\right)$ of two-point and three-point functions of stress tensors. As a consistency check, these shape coefficients $f_{a}$ and $f_{c}$ satisfy the differential relation as derived previously for the Rényi entropy. Interestingly, these holographic relations also apply to weakly coupled conformal field theories such as theories of free fermions and vectors but are violated by theories of free scalars. The mismatch of $f_{a}$ for scalars has been observed in the literature and is due to certain delicate boundary contributions to the modular Hamiltonian. Interestingly, we find a combination of our holographic relations which are satisfied by all free CFTs including scalars. We conjecture that this combined relation is universal for general CFTs in four dimensional spacetime. Finally, we find there are similar universal laws for holographic Rényi entropy in general dimensions.

Keywords: AdS-CFT Correspondence, Classical Theories of Gravity, Conformal Field Theory

ARXIV EPRINT: 1608.00328 


\section{Contents}

1 Introduction 1

2 Holographic Rényi entropy for higher derivative gravity 5

2.1 Gauss-Bonnet gravity 5

$\begin{array}{lll}2.1 .1 & f_{a}(n) & 6\end{array}$

$\begin{array}{lll}2.1 .2 & f_{c}(n) & 8\end{array}$

$\begin{array}{llr}2.1 .3 & f_{b}(n) & 9\end{array}$

2.2 General higher curvature gravity 11

$\begin{array}{lll}2.2 .1 & f_{a}(n) & 14\end{array}$

$\begin{array}{lll}2.2 .2 f_{c}(n) & 16\end{array}$

$\begin{array}{lll}2.2 .3 & f_{b}(n) & 16\end{array}$

$\begin{array}{lll}3 & \text { The story of free CFTs } & 17\end{array}$

4 Universality of HRE in general dimensions $\quad 18$

$\begin{array}{lll}4.1 & \text { CFTs in three dimensions } & 21\end{array}$

$\begin{array}{lll}4.2 & \text { CFTs in higher dimensions } & 22\end{array}$

5 Conclusions $\quad 26$

$\begin{array}{ll}\text { A Equivalence between two stress tensors } & 26\end{array}$

$\begin{array}{ll}\text { B Solutions in general higher curvature gravity } & 28\end{array}$

C Universal laws in general dimensions $\quad 30$

\section{Introduction}

One of the most mysterious features of quantum mechanics is the phenomena of entanglement. For system described by a density matrix $\rho$, entanglement can be conveniently measured in terms of the entanglement entropy and the Rényi entropy

$$
\begin{aligned}
S_{E E} & =-\operatorname{Tr}(\rho \log \rho), \\
S_{n} & =\frac{1}{1-n} \log \operatorname{Tr}\left(\rho^{n}\right) .
\end{aligned}
$$

For any integer $n>1$, the Rényi entropy $S_{n}$ may be obtained from

$$
S_{n}=\frac{\log Z_{n}-n \log Z_{1}}{1-n}
$$


where $Z_{n}$ is the partition function of the field theory on a certain $n$-fold branched cover manifold. The Rényi entropy provides a one parameter family of entanglememt measurement labeled by an integer $n$, from which entanglement entropy $S_{E E}$ can be obtained as a limit

$$
S_{E E}=\lim _{n \rightarrow 1} S_{n}
$$

if $S_{n}$ is continued to real $n$.

The study of entanglement entropy and the nature of quantum nonlocality has brought new insights into our understandings of gravity. It is found that entanglement plays an important role in the emergence of space-time and gravitational dynamics [1-5]. In addition to entanglement entropy, Rényi entropy has drawn much attention recently, including the holographic formula of Rényi entropy [6, 7], the shape dependence of Rényi entropy [8-10], the holographic dual of boundary cones [11] and Rényi twist displacement operator $[12,13]$.

Generally, for a spatial region $A$ in a $d$-dimensional spacetime, the Rényi entropy for $A$ is UV divergent. If one organizes in terms of the short distance cutoff $\epsilon$, one finds it contain a universal term in the sense that it is independent on the UV regularization scheme one choose. In odd spacetime dimensions, the universal term is $\epsilon$ independent. In even spacetime dimensions, the universal term is proportional to $\log \epsilon$ and its coefficient can be written in terms of geometric invariant of the entangling surface $\Sigma=\partial A$. In four dimensions, the universal term of the Rényi entropy has the following geometric expansion [14, 15],

$$
S_{n}^{\text {univ }}=\log \epsilon\left(\frac{f_{a}(n)}{2 \pi} \mathcal{R}_{\Sigma}+\frac{f_{b}(n)}{2 \pi} \mathcal{K}_{\Sigma}-\frac{f_{c}(n)}{2 \pi} \mathcal{C}_{\Sigma}\right)
$$

Here the conformal invariants are

$$
\mathcal{R}_{\Sigma} \equiv \int_{\Sigma} d^{2} y \sqrt{\sigma} R_{\Sigma}, \quad \mathcal{C}_{\Sigma} \equiv \int_{\Sigma} d^{2} y \sqrt{\sigma} C^{a b}{ }_{a b}, \quad \mathcal{K}_{\Sigma} \equiv \int_{\Sigma} d^{2} y \sqrt{\sigma} \operatorname{tr}\left(\bar{K}^{2}\right)
$$

where $\sigma, R_{\Sigma}, \bar{K}_{\hat{i} \hat{j}}, C^{a b}{ }_{a b}$ are, respectively, the induced metric, intrinsic Ricci scalar, trace-less part of extrinsic curvature and the contraction of the Weyl tensor projected to directions orthogonal to the entangling surface $\Sigma$. The shape dependence of the Rényi entropy is described by the coefficients $f_{a}, f_{b}, f_{c}$, which depend on $n$ and the details of CFTs in general. The coefficient $f_{a}$ can be obtained by studying the thermal free energy of CFTs on a hyperboloid [6]. The coefficients $f_{c}$ and $f_{b}$ are determined by the stress tensor onepoint function and two-point function on the hyperboloid background $[12,16]$. Remarkably, it is found in [16] that $f_{c}$ is completely determined by $f_{a}$ :

$$
f_{c}(n)=\frac{n}{n-1}\left[f_{a}(1)-f_{a}(n)-(n-1) f_{a}^{\prime}(n)\right] .
$$

It was conjectured in [17] that

$$
f_{b}(n)=f_{c}(n)
$$

holds for general 4d CFTs. This conjecture has passed numerical test for free scalar and free fermion [17]. According to [12], it seems that the relation (1.8) holds only for free CFTs. Evidence includes an analytic proof for free scalar. However, it is found to be violated by strongly coupled CFTs with Einstein gravity duals [9]. 
In this paper, we apply the holographic approach developed in $[9,10,13]$ to study the universal terms of the Rényi entropy for CFTs in general spacetime dimensions that admit general higher derivative gravity duals. For $4 \mathrm{~d}$ CFTs, expanding the coefficients $\left(f_{a}, f_{b}, f_{c}\right)$ in powers of $(n-1)$, we find the leading and sub-leading terms are related to parameters $\left(c, t_{2}, t_{4}\right)$ of two point and three point functions of stress tensors $[18,19]$ :

$$
\begin{aligned}
& f_{a}(n)=a-\frac{c}{2}(n-1)+c\left(\frac{35}{54}+\frac{7}{324} t_{2}+\frac{1}{81} t_{4}\right)(n-1)^{2}+O(n-1)^{3} \\
& f_{b}(n)=c-c\left(\frac{11}{12}+\frac{1}{18} t_{2}+\frac{1}{45} t_{4}\right)(n-1)+O(n-1)^{2} \\
& f_{c}(n)=c-c\left(\frac{17}{18}+\frac{7}{108} t_{2}+\frac{1}{27} t_{4}\right)(n-1)+O(n-1)^{2} .
\end{aligned}
$$

It should be mentioned that the expansion (1.9) of $f_{a}$ has been obtained in [20] by using twopoint and three-point function of the modular Hamiltonian. Here we provide a holographic proof of it. We note that (1.9) and (1.11) satisfy the relation (1.7). This can be regarded as a check of our holographic calculations. We also note that $t_{2}=t_{4}=0$ for Einstein gravity and the eqs. (1.10), (1.11) reduce to the results obtained in [9] in this case. To the best of our knowledge, the universal dependence of $f_{b}$ on the coefficients $t_{2}, t_{4}$ as obtained in the relation (1.10) is new. This is one of the main results of this paper.

We remark that our holographic relations eqs. (1.9)-(1.11) are also satisfied by free fermions and vectors. ${ }^{1}$ However, mismatch appears for free scalars. Actually, the discrepancy of $f_{a}$ in scalars has been observed in [20], which is due to the boundary contributions to the modular Hamiltonian. It was found that the boundary terms in the stress tensor of scalars are important at weak coupling and are suppressed in the strong coupling limit [20]. Although eqs. (1.9), (1.10), (1.11) are not satisfied by theories of free scalars, we find that the following combinations

$$
\begin{aligned}
2 f_{b}^{\prime}(1)-3 f_{c}^{\prime}(1) & =c\left(1+\frac{1}{12} t_{2}+\frac{1}{15} t_{4}\right), \\
2 f_{b}^{\prime}(1)+\frac{9}{2} f_{a}^{\prime \prime}(1) & =c\left(4+\frac{1}{12} t_{2}+\frac{1}{15} t_{4}\right),
\end{aligned}
$$

are satisfied by all CFTs with holographic dual and all free CFTs including free scalars. We conjecture they are universal relations for all CFTs in four dimensions. Note that we have $f_{c}^{\prime}(1)+\frac{3}{2} f_{a}^{\prime \prime}(1)=c$ from eq. (1.7), therefore eq. (1.12) and eq. (1.13) are not independent. Without loss of generality, we focus on the conjecture eq. (1.12) in the rest of this paper.

In the notation of [12], our conjecture (1.12) for 4d CFTs can be written in the form

$$
\pi C_{D}^{\prime \prime}(1)-36 h_{n}^{\prime \prime}(1)=\frac{2 \pi^{3}}{5} C_{T}\left(1+\frac{1}{12} t_{2}+\frac{1}{15} t_{4}\right)
$$

where $C_{T}=\frac{40}{\pi^{4}} c, h_{n}(n)$ and $C_{D}(n)$ are CFT data associated with the presence of the entangling surface. In general, for a $d$-dimensional CFT and an entangling surface $\Sigma$

\footnotetext{
${ }^{1}$ We have assume $f_{b}^{\prime \prime}(1)=f_{c}^{\prime \prime}(1)$ for free fermions and vectors. Numerical calculations support this assumption for free fermions [17].
} 
(codimension 2), one denotes the coordinates orthogonal and parallel to the entangling surface by $x^{a}$ and $y^{\hat{i}}$. The breaking of translational invariance in the directions transverse to $\Sigma$ can be characterized by the displacement operators $D^{a}\left(y^{\hat{i}}\right)$. As a result, one has the following correlation functions [12]:

$$
\begin{aligned}
\left\langle T_{\hat{i} \hat{j}}\right\rangle_{n} & =-\frac{h_{n}(n)}{2 \pi n} \frac{\delta^{\hat{i} j}}{\left|x^{a}\right|^{d}}, \\
\left\langle D^{a}\left(y^{\hat{i}}\right) D^{b}(0)\right\rangle_{n} & =C_{D}(n) \frac{\delta^{a b}}{\left|y^{\hat{i}}\right|^{2(d-1)}} .
\end{aligned}
$$

Here $h_{n}(n)$ is the coefficient fixing the normalization of the one-point function for the stress tensor in the presence of the twisted operator for the $n$-fold replicated QFT, and $C_{D}(n)$ is the normalization coefficient for the two-points correlation function of the displacement operators. In 4-dimensions, $C_{D}(n)$ and $h_{n}(n)$ related to the dependence of Rényi and entanglement entropy on smooth or shape deformations [8,21-23]. The specific relation can be found in eqs. (2.12), (3.15), (3.19) of [12].

It should be mentioned that unlike $f_{c}$ and $f_{b}$ which are defined only in 4 dimensions, $h_{n}$ and $C_{D}$ have a natural definition in all dimensions. Therefore it is natural to ask if by using them one can generalize the results (1.12) and (1.13) to other dimensional spacetime. The holographic dual of $h_{n}$ and $C_{D}$ for Einstein gravity and Gauss-Bonnet Gravity in general dimensions are studied in recent works $[10,13]$. Applying their results, one can express $h_{n}^{\prime \prime}(1)$ and $C_{D}^{\prime \prime}(1)$ in terms of $C_{T}$ and $t_{2}$. Recall that we have $t_{4}=0$ for Einstein gravity and Gauss-Bonnet Gravity. To get the information of $t_{4}$, one has to study at least one cubic curvature term such as $\mathcal{K}_{7}$ and $\mathcal{K}_{8}$ in the action (2.43). Following the approach of $[10,13]$, we obtain the holographic formulae of $h_{n}$ and $C_{D}$ for a $d$-dimensional CFT admiting a general higher curvature gravity dual:

$$
\begin{aligned}
& \frac{h_{n}}{C_{T}}=-2 \pi n \frac{M_{e}}{f_{d}}, \\
& \frac{C_{D}}{C_{T}}=\frac{d \pi^{2} n}{d+1}\left[(d-2)\left(\beta_{n}-\beta_{1}\right)-\frac{M_{e}}{2}\right],
\end{aligned}
$$

where $M_{e}$ is the effective mass defined in eq. (4.14) and $\beta_{n}$ is the coefficient in the function $k(r)$ in eq.(4.15) which describes a deformation in the extrinsic curvature of the entangling surface. It is remarkable that these relations take simple and universal form for all the higher curvature gravity.

By using the holographic formula of $h_{n}$ and $C_{D}$, we find there are similar universal laws in general dimensions, which involves linear combinations of the terms $C_{D}^{\prime \prime}(1), h_{n}^{\prime \prime}(1), C_{T}, C_{T} t_{2}$ and $C_{T} t_{4} \cdot{ }^{2}$ In general, we have for a $d$-dimensional CFT,

$$
\begin{aligned}
\frac{h_{n}^{\prime \prime}(1)}{C_{T}}= & -\frac{2 \pi^{\frac{d}{2}+1} \Gamma\left(\frac{d}{2}\right)}{(d-1)^{3} d(d+1) \Gamma(d+3)}\left[d\left(2 d^{5}-9 d^{3}+2 d^{2}+7 d-2\right)\right. \\
& \left.+(d-2)(d-3)(d+1)(d+2)(2 d-1) t_{2}+(d-2)\left(7 d^{3}-19 d^{2}-8 d+8\right) t_{4}\right] \\
\frac{C_{D}^{\prime \prime}(1)}{C_{T}}= & \frac{4 \pi^{2}}{d+1}\left[\frac{1-d^{2}+d}{d^{2}-d}-\frac{(d-2)(d-3)}{(d-1)^{2} d} t_{2}-\frac{(d-2)\left(3 d^{2}-7 d-8\right)}{(d-1)^{2} d(d+1)(d+2)} t_{4}\right] .
\end{aligned}
$$

\footnotetext{
${ }^{2}$ In three dimensions, we have $t_{2}=0$. And we have $t_{2}=t_{4}=0$ in two dimensions.
} 
Note that the relation $C_{D}^{\prime \prime}(1)=d \Gamma\left(\frac{d+1}{2}\right)\left(\frac{2}{\sqrt{\pi}}\right)^{d-1} h_{n}^{\prime \prime}(1)$ is obeyed by free fermions and conformal tensor fields ${ }^{3}$ but are violated by free scalars. However similar to the 4 dimensional case, there exist 'universal laws' that include free scalar fields. For example, in three dimensions, we find

$$
\pi C_{D}^{\prime \prime}(1)-16 h_{n}^{\prime \prime}(1)=\frac{\pi^{3}}{3} C_{T}\left(1+\frac{t_{4}}{30}\right)
$$

works well for free fermions, free scalars and CFTs with gravity dual. As for the 'universal laws' in higher dimensions, please refer to eq. (4.48). It is interesting to study whether these 'universal laws' are obeyed by more general CFTs.

The paper is organized as follows. In section2, we study 4d CFTs which are dual to general higher curvature gravity and derive the relations between the coefficients $\left(f_{a}^{\prime \prime}(1), f_{b}^{\prime}(1), f_{c}^{\prime}(1)\right)$ in the universal terms of Rényi entropy and the parameters $\left(c, t_{2}, t_{4}\right)$ of two point and three point functions of the stress tensors in the conformal field theory. In section 3, we compare these holographic relations with those of free CFTs and find a combined relation which agrees with all the known results of the free CFTs. We conjecture this combined relation is a universal law for all the CFTs in four dimensions. In section 4, we consider three and higher general spacetime dimensions and derive the holographic dual of $h_{n}$ and $C_{D}$ for general higher curvature gravity and discuss the universal behaves of $h_{n}^{\prime \prime}(1)$ and $C_{D}^{\prime \prime}(1)$. Finally, we conclude in section5.

Notations: we use $x^{\mu}\left(y^{i}\right)$ and $g_{\mu \nu}\left(\gamma_{i j}\right)$ to denote the coordinates and metric in the bulk (on the boundary). $x^{a}$ and $y^{\hat{i}}$ are the orthogonal and parallel coordinates on the entangling surface. $\sigma_{\hat{i} \hat{j}}$ is the induced metric on the entangling surface. For simplicity, we focus on Euclidean signature in this paper.

\section{Holographic Rényi entropy for higher derivative gravity}

In this section, we investigate the universal terms of Rényi entropy for $4 \mathrm{~d}$ CFTs that are dual to general higher derivative gravity. We firstly take Gauss-Bonnet gravity as an example and then generalize the results to general higher curvature gravity. Some interesting relations between the universal terms of holographic Rényi entropy (HRE) and the parameters of two point and three point functions of stress tensors are found.

\subsection{Gauss-Bonnet gravity}

For simplicity, we consider the following Gauss-Bonnet Gravity which is slightly different from the standard form

$$
I=\frac{1}{16 \pi G_{N}} \int_{M}\left[R+\frac{12}{l^{2}}+\alpha\left(\bar{R}_{\mu \nu \rho \sigma} \bar{R}^{\mu \nu \rho \sigma}-4 \bar{R}_{\mu \nu} \bar{R}^{\mu \nu}+\bar{R}^{2}\right)\right]+I_{B}
$$

\footnotetext{
${ }^{3}$ The conformal tensor fields appear only in even dimensions.
} 
where $\int_{M} \equiv \int_{M} d^{d+1} x \sqrt{g}(d=4$ here $)$, the quantities $\bar{R}_{\ldots}^{\cdots}$ are given by

$$
\begin{aligned}
\bar{R}_{\mu \nu \rho \sigma} & =R_{\mu \nu \rho \sigma}-\frac{1}{l^{2}}\left(g_{\mu \sigma} g_{\nu \rho}-g_{\mu \rho} g_{\nu \sigma}\right), \\
\bar{R}_{\mu \nu} & =R_{\mu \nu}+\frac{4}{l^{2}} g_{\mu \nu}, \\
\bar{R} & =R+\frac{20}{l^{2}}
\end{aligned}
$$

and $I_{B}$ denotes the Gibbons-Hawking-York terms which make a well-defined variational principle and the counter terms which make the total action finite.

An advantage of the above action is that, similar to Einstein gravity, the radius of AdS is exactly $l$. While in the standard GB and higher derivative gravity, the effective radius of AdS is a complicated function of $l$, which makes the calculations complicated. Below we set $l=1$ for simplicity.

\subsection{1 $f_{a}(n)$}

Let us briefly review the method to derive $f_{a}(n)[6]$. We focus on the spherical entangling surface, where $\operatorname{tr} \bar{K}^{2}$ and $C_{a b}^{a b}$ vanish. Thus only $f_{a}$ appears in the universal terms of Rényi entropy eq. (1.5). The main idea is to map the vacuum state of the CFTs in a spherical entangling region to the thermal state of CFTs on a hyperboloid. The later has a natural holographic dual in the bulk, the black hole that asymptotes to the hyperboloid. Using the free energy of black hole, we can derive Rényi entropy as

$$
S_{n}=\frac{n}{1-n} \frac{1}{T_{0}}\left[F\left(T_{0}\right)-F\left(T_{0} / n\right)\right]
$$

where $T_{0}$ is the temperature of hyperbolic black hole for $n=1$. Further using the thermodynamic identity, $S=-\partial F / \partial T$, we can rewrite the above expression as

$$
S_{n}=\frac{n}{n-1} \frac{1}{T_{0}} \int_{T_{0} / n}^{T_{0}} S_{\mathrm{BH}}(T) d T
$$

where $S_{\mathrm{BH}}(T)$ is the black hole entropy. For our revised GB gravity (2.1), it takes the form

$$
S_{\mathrm{BH}}=\frac{1}{4 G_{N}} \int_{H} d y^{3} \sqrt{h}\left[1+2 \alpha\left(\mathcal{R}_{H}+6\right)\right]
$$

where $H$ denotes horizon and $\mathcal{R}_{H}$ is the intrinsic Ricci scalar on horizon.

The key point in this approach is finding the black hole solution that asymptotes to the hyperboloid on the boundary. We get

$$
d s_{\text {bulk }}^{2}=\frac{d r^{2}}{f(r)}+f(r) d \tau^{2}+r^{2} d \Sigma_{3}^{2}
$$

where $d \Sigma_{3}^{2}$ is the line element for hyperbolic plane $H^{3}$ with unit curvature, and $f(r)$ is given by

$$
f(r)=\frac{(1+12 \alpha) r^{2}-\sqrt{8 \alpha M+(1+8 \alpha)^{2} r^{4}}}{4 \alpha}-1
$$


Here

$$
M=\left(r_{H}^{2}-1\right)\left((1+10 \alpha) r_{H}^{2}-2 \alpha\right),
$$

and $r_{H}$ denotes the position of horizon, $f\left(r_{H}\right)=0$. Note that $f(r)$ has the correct limit: it becomes that of hyperbolic black hole (black hole in Einstein gravity) when $M \rightarrow 0$ $(\alpha \rightarrow 0)$. In the large $r$ limit, the boundary metric is conformal equivalent to

$$
d s_{\text {boun }}^{2}=d \tau^{2}+d \Sigma_{3}^{2}
$$

which is the expected metric on manifold $S^{1} \times H^{3}$.

To determine $r_{H}$, we note that the Hawking temperature on horizon is given by

$$
T=\left.\frac{1}{4 \pi} \partial_{r} f(r)\right|_{r=r_{H}}=\frac{1}{2 \pi n} .
$$

From the above equation, one can easily get $T_{0}=\frac{1}{2 \pi}$ for the hyperbolic black hole with $f(r)=r^{2}-1$ and $r_{H}=1$. Now let us solve eq. (2.12) and express $r_{H}$ in terms of $(n-1)$

$$
r_{H}=1-\frac{n-1}{3}+\frac{(10+96 \alpha)}{27(1+8 \alpha)}(n-1)^{2}-\frac{2\left(49+912 \alpha+4224 \alpha^{2}\right)}{243(1+8 \alpha)^{2}}(n-1)^{3}+O(n-1)^{4} .
$$

Substituting eq. (2.13) together with $T=\frac{1}{2 \pi n}, T_{0}=\frac{1}{2 \pi}$ and $\mathcal{R}_{H}=-6 / r_{H}^{2}$ into eqs. (2.6), (2.7), we obtain

$$
\begin{aligned}
S_{n}= & \frac{V_{\Sigma}}{4 G_{N}}\left[1-\frac{1+8 \alpha}{2}(n-1)+\frac{7}{54}(5+48 \alpha)(n-1)^{2}-\frac{\left(11856 \alpha^{2}+2514 \alpha+133\right)}{162(8 \alpha+1)}(n-1)^{3}\right] \\
& +O(n-1)^{4},
\end{aligned}
$$

where $V_{\Sigma}$ is the hyperbolic volume, which contributes a logarithmic term $V_{\Sigma}^{\text {univ }}=$ $2 \pi \log \epsilon[6]$. Now we can extract $f_{a}$ from eq. (2.14) as

$$
\begin{aligned}
f_{a}(n) & =a-\frac{c}{2}(n-1)+\frac{7}{54}(6 c-a)(n-1)^{2}+O(n-1)^{3} \\
& =a-\frac{c}{2}(n-1)+c\left(\frac{35}{54}+\frac{7}{324} t_{2}\right)(n-1)^{2}+O(n-1)^{3},
\end{aligned}
$$

where $a=\frac{\pi}{8 G_{N}}$ and $c=\frac{\pi}{8 G_{N}}(1+8 \alpha)[24]$. In the above derivation we have used

$$
\frac{c-a}{c}=\frac{1}{6} t_{2}+\frac{4}{45} t_{4}, \quad \text { for } 4 \mathrm{~d} \text { CFTs }
$$

and $t_{4}=0$ for GB gravity. Clearly, eq. (2.15) agrees with eq. (1.9) when $t_{4}=0$. To get the information of $t_{4}$, one must consider more general higher derivative gravity. We leave this problem to next section. Notice that the $O(n-1)^{3}$ terms of $f_{a}$ eq. (2.14) is a complicated function of $a$ and $c$, which implies that there is no universal relations at this and higher orders. From the viewpoint of CFTs, terms of $f_{a}$ at order $O(n-1)^{3}$ are determined by fourpoint functions of stress tensor [20]. Unlike two-point and three-point functions, four-point functions of CFTs are no longer universal. Thus, it is expected that there is no universal relation at order $O(n-1)^{3}$ for $f_{a}$. It depends on the details of CFTs at this and higher orders. Similarly, one expects there is no universal relation at order $O(n-1)^{2}$ for $f_{b}$ and $f_{c}$. 


\subsection{2 $f_{c}(n)$}

Now let us continue to derive $f_{c}(n)$. We take the approach developed in [9]. In general with a deformation of the field theory metric, the change in the partition function is govern by one-point function of the field theory stress tensor

$$
\delta \log Z_{n}=\frac{1}{2} \int_{\partial M} d x^{4} \sqrt{\gamma}\left\langle T^{i j}\right\rangle \delta \gamma_{i j}
$$

The main idea of [9] is to consider specific deformation of the metric so that, on using (1.3), one may isolate the required shape dependent term in the universal part of the Rényi entropy. For example, $f_{c}$ can be isolated with a deformation that affects $\mathcal{C}_{\Sigma}$ but not $\mathcal{K}_{\Sigma}$ :

$$
\delta S_{n}=-\log \epsilon \int_{\Sigma} d^{2} y \sqrt{\sigma} \frac{f_{c}(n)}{2 \pi} C^{a b}{ }_{a b}+\cdots
$$

where $\cdots$ are non-universal terms of the Rényi entropy. This can be achieved by considering on the entangling surface the following metric

$$
d s_{\text {boun }}^{2}=d \tau^{2}+\frac{1}{\rho^{2}}\left[d \rho^{2}+\left(\delta_{\hat{i} \hat{j}}+Q_{a b \hat{i} \hat{j}} x^{a} x^{b}+O\left(\rho^{3}\right)\right) d y^{\hat{i}} d y^{\hat{j}}\right]
$$

where $Q_{a b \hat{i} \hat{j}}$ describes a deformation of the metric and give rises to an amount of $C^{a b}{ }_{a b}$ as

$$
C^{a b}{ }_{a b}=\frac{1}{3} Q_{a}{ }^{a \hat{i}} \hat{i} .
$$

Here in (2.19), we have adopted a local coordinate system $\left(\rho, \tau, y^{\hat{i}}\right)$ near $\Sigma$, where for each point on $\Sigma$, we introduce a one-parameter family of geodesics orthogonal to $\Sigma$ parametrized by $\tau$, and $\rho$ denotes the radial distance to $\Sigma$ along such a geodesic. $\left(x^{1}, x^{2}\right) \equiv(\rho \cos \tau, \rho \sin \tau)$ and $\left\{y^{\hat{i}}, i=1, \cdots, d-2\right\}$ denotes an arbitrary coordinates system on $\Sigma$. We note that in this computation of $f_{c}$, the boundary metric (2.19) is conformal equivalent to a deformed conical metric.

To proceed with the calculation of $f_{c}(n)$, we consider the bulk metric that asymptotes to the deformed hyperboloid background (2.19):

$$
d s_{\text {bulk }}^{2}=\frac{d r^{2}}{f(r)}+f(r) d \tau^{2}+\frac{r^{2}}{\rho^{2}}\left[d \rho^{2}+\left(\delta_{\hat{i} \hat{j}}+q(r) Q_{a b \hat{i} \hat{j}} x^{a} x^{b}+O\left(\rho^{3}\right)\right) d y^{\hat{i}} d y^{\hat{j}}\right]
$$

where $q(r)$ is determined by the E.O.M in the bulk and approach 1 in the limit $r \rightarrow \infty$. Actually, to derive $f_{c}(n)$, we do not need to solve the E.O.M. That is because we already have $\delta \gamma_{i j}=\frac{r^{2}}{\rho^{2}} Q_{a b \hat{i} j} x^{a} x^{b} \sim C_{a b}^{a b}$, so we only need zero order of $T^{i j}$ in eq. (2.17) in order to extract the terms proportional to $C_{a b}^{a b}$. In other words, we only need to calculate $T^{i j}$ on undeformed hyperboloid background.

We note that in the context of AdS/CFT, the stress tensor that appears in (2.17) can be taken either as the regularized Brown-York boundary stress tensor [25] or the holographic stress tensor [26]. The two are equivalent as we demonstrate in the appendix. In this section, we will consider the first approach. The key point is to find the regularized boundary stress tensor for our non-standard GB gravity (2.1). Notice that our non-standard GB 
gravity (2.1) can be rewritten into the standard form, with only the coefficients of $\mathcal{L}_{0}=1$ and $\mathcal{L}_{2}=R$ different from the standard GB:

$$
\begin{aligned}
I & =\frac{1}{16 \pi G_{N}} \int_{M}\left[R+\frac{d^{2}-d}{l^{2}}+\alpha \mathcal{L}_{4}(\bar{R})\right], \\
& =\frac{1}{16 \pi G_{N}} \int_{M}\left[(1+2(d-1)(d-2) \alpha) R+\frac{d^{2}-d}{l^{2}}(1+(d+1)(d-2) \alpha)+\alpha \mathcal{L}_{4}(R)\right],
\end{aligned}
$$

where $\mathcal{L}_{4}(R)$ denotes the standard GB term. The holographic regularization for GB gravity is studied in [27]. Reparameterizing their formulas, we get for the Brown-York boundary stress tensor:

$$
\begin{aligned}
8 \pi G_{N} T_{\partial M}^{i j}= & (1+2 \alpha(d-1)(d-2))\left[K_{\partial M}^{i j}-K_{\partial M} \gamma^{i j}-(d-1) \gamma^{i j}+\frac{\Theta(d-3)}{d-2}\left(R_{\partial M}^{i j}-\frac{1}{2} R_{\partial M} \gamma^{i j}\right)\right] \\
& +2 \alpha\left(Q^{i j}-\frac{1}{3} Q \gamma^{i j}\right)
\end{aligned}
$$

where $\Theta(x)$ is the step-function with $\Theta(x)=1$ provided $x \geq 0$, and zero otherwise. $K_{\partial M}^{i j}$ is the extrinsic curvature on the AdS boundary and $Q_{i j}$ is given by

$$
\begin{aligned}
Q_{i j}= & 2 K_{\partial M} K_{\partial M i k} K_{\partial M}{ }_{j}-2 K_{\partial M i k} K_{\partial M}^{k l} K_{\partial M l j}+K_{\partial M i j}\left(K_{\partial M k l} K_{\partial M}^{k l}-K_{\partial M}^{2}\right) \\
& +2 K_{\partial M} R_{\partial M i j}+R_{\partial M} K_{\partial M i j}-2 K_{\partial M}^{k l} R_{\partial M k i l j}-4 R_{\partial M i k} K_{\partial M}^{k}{ }_{j} .
\end{aligned}
$$

Here $R_{\partial M}$ denotes the intrinsic curvature on the boundary.

Substituting eq. (2.23) and $\delta \gamma_{i j}=\frac{r^{2}}{\rho^{2}} Q_{a b \hat{i} \hat{j}} x^{a} x^{b}$ into eqs. (2.17), (2.18), we obtain

$$
\begin{aligned}
f_{c}(n) & =\frac{\pi}{8 G_{N}}\left[1+8 \alpha+\left(-\frac{17}{18}-\frac{32 \alpha}{3}\right)(n-1)+\frac{217+4512 \alpha+23232 \alpha^{2}}{162(1+8 \alpha)}(n-1)^{2}\right]+O(n-1)^{3} \\
& =c+\left(\frac{7}{18} a-\frac{4}{3} c\right)(n-1)+O(n-1)^{2} \\
& =c+c\left(-\frac{17}{18}-\frac{7}{108} t_{2}\right)(n-1)+O(n-1)^{2}
\end{aligned}
$$

Similar to $f_{a}(n)$, we have used $\frac{c-a}{c}=\frac{1}{6} t_{2}+\frac{4}{45} t_{4}$ for 4 d CFTs and $t_{4}=0$ for GB gravity. Eq. (2.25) agrees with eq. (1.11) when $t_{4}=0$. Note that eq. (2.25) and eq. (2.15) are consistent with the identity (1.7). This can be regarded as a check of our holographic calculations.

\subsection{3 $f_{b}(n)$}

Now let us go on to calculate $f_{b}(n)$. The method is similar to that of $f_{c}(n)$ : we consider the first order variation (2.17) of the partition on the hyperboloid background deformed by an extrinsic curvature [9] and then extract $f_{b}(n)$ from

$$
\delta S_{n}=\log \epsilon \int_{\Sigma} d^{2} y \sqrt{\sigma} \frac{f_{b}(n)}{2 \pi} \operatorname{tr}\left(\bar{K}^{2}\right)+\cdots .
$$

The main difference from $f_{c}(n)$ is that now we need to calculate $T^{i j}$ on the deformed hyperboloid $\tilde{H}_{n}^{4}$. This is because we have $\delta \gamma_{i j} \sim K$, thus to extract $K^{2}$ terms, we must get $T^{i j}$ of order $K$. 
To proceed, we deform the boundary hyperboloid by a traceless extrinsic curvature

$$
d s_{\text {boun }}^{2}=d \tau^{2}+\frac{1}{\rho^{2}}\left[d \rho^{2}+\left(\delta_{\hat{i} \hat{j}}+K_{a \hat{i} \hat{j}} x^{a}+O\left(\rho^{2}\right)\right) d y^{\hat{i}} d y^{\hat{j}}\right] .
$$

Then the bulk metric becomes

$$
d s_{\text {bulk }}^{2}=\frac{d r^{2}}{f(r)}+f(r) d \tau^{2}+\frac{r^{2}}{\rho^{2}}\left[d \rho^{2}+\left(\delta_{\hat{i} \hat{j}}+k(r) K_{a \hat{i} \hat{j}} x^{a}+O\left(\rho^{2}\right)\right) d y^{\hat{i}} d y^{\hat{j}}\right]
$$

To get boundary stress tensor $T^{i j}$ of order $O(K)$, we need to solve the E.O.M up to $O(K)$. For traceless $K_{a i j}$, we find one independent equation

$$
\begin{aligned}
& {\left[-2 \alpha\left(f f^{\prime \prime}+r f^{\prime}-6\left(f+r^{2}\right)\right)+f+r^{2}\right] k(r)} \\
& +f\left[r f\left(-36 \alpha+2 \alpha f^{\prime \prime}-3\right)-f^{\prime}\left((12 \alpha+1) r^{2}-4 \alpha f\right)+2 \alpha r\left(f^{\prime}\right)^{2}\right] k^{\prime}(r) \\
& -r f^{2}\left(-2 \alpha f^{\prime}+12 \alpha r+r\right) k^{\prime \prime}(r)=0
\end{aligned}
$$

Near the horizon, the solutions behave like $k(r) \sim\left(r-r_{H}\right)^{n / 2}$. The solution is uniquely determined by this IR boundary boundary condition and the UV boundary condition $\lim _{r \rightarrow \infty} k(r)=1$. However, the IR boundary boundary condition $k(r) \sim\left(r-r_{H}\right)^{n / 2}$ is not easy to deal with. Thus, we define a new function

$$
h(r)=k(r) \exp \left[\int_{r}^{\infty} \frac{d r}{f(r)}\right]
$$

and the E.O.M becomes

$$
\begin{aligned}
& {\left[2 \alpha(r-1) f^{\prime \prime}+4 \alpha f^{\prime}+(12 \alpha+1)(-(3 r-1))\right] h(r)} \\
& +\left[f\left(r\left(-36 \alpha+2 \alpha f^{\prime \prime}-3\right)+4 \alpha f^{\prime}\right)-r\left(f^{\prime}+2\right)\left(-2 \alpha f^{\prime}+12 \alpha r+r\right)\right] h^{\prime}(r) \\
& +\left[-f r\left(-2 \alpha f^{\prime}+12 \alpha r+r\right)\right] h^{\prime \prime}(r)=0
\end{aligned}
$$

Now the regularity condition at horizon simply requires $h\left(r_{H}\right)$ to be finite. Solving the above equation perturbatively, we get

$$
h(r)=\frac{r+1}{r}+h_{1}(r)(n-1)+h_{2}(r)(n-1)^{2}+\cdots
$$

with

$$
\begin{aligned}
h_{1}(r)= & \frac{r+1}{r} \log \left(\frac{r+1}{r}\right)-\frac{6 r^{2}+3 r-1}{6 r^{3}}, \\
h_{2}(r)= & \frac{r+1}{2 r} \log ^{2}\left(\frac{r+1}{r}\right)-\frac{6 r^{2}+3 r-1}{6 r^{3}} \log \left(\frac{r+1}{r}\right) \\
& +\frac{5\left(216 r^{3}-85 r+27\right) r^{2}+24\left(r\left(r\left(360 r^{3}-155 r+69\right)+4\right)+20\right) \alpha}{2160 r^{7}(1+8 \alpha)}, \\
& \cdots
\end{aligned}
$$

where we have obtained solutions up to $h_{5}(r)$. For simplicity we do not list them here. 
From eqs. (2.30), (2.32), (2.33), we can derive $k(r)$. Expanding $k(r)$ in large $r$, we find

$$
k(r)=1-\frac{1}{2 r^{2}}+\frac{\beta_{n}}{r^{4}}+O\left(\frac{1}{r^{6}}\right)
$$

where

$$
\begin{aligned}
& \beta_{n}=-\frac{1}{8}+\frac{n-1}{12}-\frac{(67+600 \alpha)}{432(1+8 \alpha)}(n-1)^{2}+\frac{\left(151104 \alpha^{2}+34320 \alpha+1945\right)}{7776(1+8 \alpha)^{2}}(n-1)^{3} \\
& -\frac{\left(1362415104 \alpha^{3}+471579456 \alpha^{2}+54244296 \alpha+2074355\right)}{5598720(8 \alpha+1)^{3}}(n-1)^{4} \\
& +\frac{\left(19865723572224 \alpha^{4}+9304662564864 \alpha^{3}+1627900276608 \alpha^{2}+126143146752 \alpha+3654194425\right)}{7054387200(1+8 \alpha)^{4}}(n-1)^{5} \\
& +O(n-1)^{6} .
\end{aligned}
$$

Recall that $\delta \log Z \sim T^{i j} \delta \gamma_{i j}$ and (2.17) is calculated on the boundary with $r \rightarrow$ $\infty$. Thus, $k(r)$ in the large $r$ expansion is good enough for our purpose. Substituting eqs. (2.35), (2.28), (2.9), (2.13) into eqs. (2.23), (2.17), (2.26), we obtain

$$
\begin{aligned}
& \frac{8 G_{N}}{\pi} f_{b}(n)=(1+8 \alpha)-\left(\frac{11}{12}+10 \alpha\right)(n-1)+\frac{\left(27840 \alpha^{2}+5552 \alpha+275\right)}{216(1+8 \alpha)}(n-1)^{2} \\
& -\frac{\left(237436416 \alpha^{3}+74097984 \alpha^{2}+7667464 \alpha+263115\right)}{155520(1+8 \alpha)^{2}}(n-1)^{3} \\
& +\frac{\left(3323533971456 \alpha^{4}+1425617289216 \alpha^{3}+228089069952 \alpha^{2}+16137500288 \alpha+426115725\right)}{195955200(1+8 \alpha)^{3}}(n-1)^{4} \\
& +O(n-1)^{5} .
\end{aligned}
$$

Similar to $f_{c}(n)$, we can rewrite $f_{b}(n)$ in terms of $a$ and $c$ or $c$ and $t_{2}$. We have

$$
\begin{aligned}
f_{b}(n) & =c+\left(\frac{1}{3} a-\frac{5}{4} c\right)(n-1)+O(n-1)^{2} \\
& =c+c\left(-\frac{11}{12}-\frac{1}{18} t_{2}\right)(n-1)+O(n-1)^{2} .
\end{aligned}
$$

To end this section, we notice an interesting property of solutions to GB gravity (2.1). Expanding in $(n-1)$, we find the solutions such as $f(r)$ and $h(r)$ are exactly the same as those of Einstein gravity at the first order $(n-1)$. Differences appear only at higher orders. As we will prove in the next section, this is a universal property for general higher curvature gravity as long as we rescale the coefficient of $R$ as 1 .

\subsection{General higher curvature gravity}

In this section, by applying the methods illustrated in section2.1, we discuss the universal terms of Rényi entropy for CFTs dual to general higher curvature gravity. In general, it is difficult to find the exact black hole solutions in higher derivative gravity. Instead, we focus on perturbative solutions up to $(n-1)^{2}$. This is sufficient to derive $f_{a}$ of order $(n-1)^{2}$ and $f_{b}, f_{c}$ of order $(n-1)$. As we have argued above, it is expected that there is no universal behavior at higher orders, due to the fact that the higher orders are determined by four and higher point functions of stress tensor, which depend on the details of CFTs. 
Let us consider the general higher curvature gravity $I\left(R_{\mu \nu \rho \sigma}\right)$. We use the trick introduced in [24] to rewrite it into the form similar as eq. (2.1). This method together with $[28,29]$ is found to be useful to study the holographic Weyl anomaly and universal terms of entanglement entropy $[24,30-32] .{ }^{4}$ Firstly, we define a 'background-curvature' (we set the AdS radius $l=1$ below)

$$
\tilde{R}_{\mu \nu \sigma \rho}=g_{\mu \rho} g_{\nu \sigma}-g_{\mu \sigma} g_{\nu \rho}
$$

and denote the difference between the curvature and the 'background-curvature' by

$$
\bar{R}_{\mu \nu \sigma \rho}=R_{\mu \nu \sigma \rho}-\tilde{R}_{\mu \nu \sigma \rho} .
$$

Then we expand the action around this 'background-curvature' and get

$$
\begin{aligned}
I & =\frac{1}{16 \pi G_{N}} \int d^{d+1} x \sqrt{g} L\left(R_{\mu \nu \sigma \rho}\right) \\
& =\frac{1}{16 \pi G_{N}} \int d^{d+1} x \sqrt{g}\left[L_{0}+c_{1}^{(1)} \bar{R}+\left(c_{1}^{(2)} \mathcal{L}_{4}(\bar{R})+c_{2}^{(2)} \bar{R}_{\mu \nu} \bar{R}^{\mu \nu}+c_{3}^{(2)} \bar{R}^{2}\right)+\sum_{\mathrm{i}=1}^{8} c_{\mathrm{i}}^{(3)} \mathcal{K}_{\mathrm{i}}(\bar{R})+O\left(\bar{R}^{4}\right)\right]
\end{aligned}
$$

where $L_{0}=L\left(\tilde{R}_{\mu \nu \sigma \rho}\right)=\left.L\left(R_{\mu \nu \sigma \rho}\right)\right|_{\text {AdS }}$ is a constant defined by the Lagrangian for AdS solution, and $c_{\mathrm{i}}^{(n)}$ are constants which parametrize the higher derivatives correction to the Einstein action up to third orders in the curvature with $n$ denoting the order. Here $\mathcal{L}_{4}(\bar{R})$ denotes the GB term

$$
\mathcal{L}_{4}(\bar{R})=\bar{R}_{\mu \nu \rho \sigma} \bar{R}^{\mu \nu \rho \sigma}-4 \bar{R}_{\mu \nu} \bar{R}^{\mu \nu}+\bar{R}^{2},
$$

and $\mathcal{K}_{\mathrm{i}}(\bar{R})$ denotes the basis of third order curvature terms

$$
\begin{aligned}
\mathcal{K}_{\mathrm{i}}(\bar{R})=\{ & \bar{R}^{3}, \bar{R} \bar{R}_{\mu \nu} \bar{R}^{\mu \nu}, \bar{R} \bar{R}_{\mu \nu \rho \sigma} \bar{R}^{\mu \nu \rho \sigma}, \bar{R}_{\mu}^{\nu} \bar{R}_{\nu}^{\rho} \bar{R}_{\rho}^{\mu}, \bar{R}^{\mu \nu} \bar{R}^{\rho \sigma} \bar{R}_{\mu \rho \sigma \nu}, \bar{R}_{\mu \nu} \bar{R}^{\mu \rho \sigma \lambda} \bar{R}_{\rho \sigma \lambda}^{\nu}, \\
& \left.\bar{R}_{\mu \nu \rho \sigma} \bar{R}^{\mu \nu \lambda \chi} \bar{R}_{\lambda \chi}^{\rho \sigma}, \bar{R}_{\mu \nu \rho \sigma} \bar{R}^{\mu \lambda \chi \sigma} \bar{R}_{\lambda \chi}^{\nu}{ }^{\rho}\right\} .
\end{aligned}
$$

We require that the higher derivative gravity has an asymptotic AdS solution. This would impose a condition $c_{1}^{(1)}=-L_{0} / 2 d[24]$. Using this condition, we can rewrite the action $(2.41)$ as

$I=\frac{1}{16 \pi G_{N}} \int_{M}-\frac{L_{0}}{2 d}\left(R+d^{2}-d\right)+\left(c_{1}^{(2)} \mathcal{L}_{4}(\bar{R})+c_{2}^{(2)} \bar{R}_{\mu \nu} \bar{R}^{\mu \nu}+c_{3}^{(2)} \bar{R}^{2}\right)+\sum_{\mathrm{i}=1}^{8} c_{\mathrm{i}}^{(3)} \mathcal{K}_{\mathrm{i}}(\bar{R})+O\left(\bar{R}^{4}\right)$.

Rescaling $G_{N} \rightarrow \tilde{G}_{N}=-\frac{2 d}{L_{0}} G_{N}, c_{i}^{(n)} \rightarrow \tilde{c}_{i}^{(n)}=-\frac{2 d}{L_{0}} c_{i}^{(n)}$, we have

$I=\frac{1}{16 \pi \tilde{G}_{N}} \int_{M}\left(R+d^{2}-d\right)+\left(\tilde{c}_{1}^{(2)} \mathcal{L}_{4}(\bar{R})+\tilde{c}_{2}^{(2)} \bar{R}_{\mu \nu} \bar{R}^{\mu \nu}+\tilde{c}_{3}^{(2)} \bar{R}^{2}\right)+\sum_{\mathrm{i}=1}^{8} \tilde{c}_{\mathrm{i}}^{(3)} \mathcal{K}_{\mathrm{i}}(\bar{R})+O\left(\bar{R}^{4}\right)$.

Now it takes the form as eq. (2.1). For simplicity, we ignore the notation below. The E.O.M of the above gravity is

$$
P_{\mu}^{\alpha \rho \sigma} R_{\nu \alpha \rho \sigma}-2 \nabla^{\rho} \nabla^{\sigma} P_{\mu \rho \sigma \nu}-\frac{1}{2} L g_{\mu \nu}=0,
$$

\footnotetext{
${ }^{4}$ For recent discussions on entanglement entropy and the scale invariance, please see [33].
} 
with $P^{\mu \alpha \rho \sigma}=\partial L / \partial R_{\mu \alpha \rho \sigma}$.

A couple of remarks on action (2.45) are in order.

Firstly, it is clear the hyperbolic black hole which is locally AdS is a solution to action (2.45). That is because $\bar{R}_{\mu \nu \rho \sigma}=0$ in AdS. We are interested of two kinds of perturbations: the first one is $\delta g_{\mu \nu} \sim O(n-1)$ related to $f_{a}, f_{c}$, and the second one is $\delta g_{\mu \nu} \sim O((n-1), K)$ related to $f_{b}$. Remarkably, we have $\bar{R}_{\mu \nu \rho \sigma} \sim O\left(n-1, K^{2}\right)^{5}$ for the deformed metric (2.28).

Secondly, we are interested of the solutions up to $O(n-1)^{2}$ and $O(K)$, or equivalently, the action up to $O(n-1)^{3}$ and $O\left((n-1)^{2} K^{2}\right)$. As a result, we can drop the $O(\bar{R})^{4}$ terms in action (2.45) due to $O(\bar{R})^{4} \sim O\left((n-1)^{4},(n-1)^{3} K^{2}, \cdots\right)$. Recall that the terms of order $O\left((n-1)^{a} K^{b}\right)$ in the action contributes to terms at least of order $O\left((n-1)^{a-1} K^{b}\right)$ and $O\left((n-1)^{a} K^{b-1}\right)$ in the E.O.M.

Thirdly, at the linear order in $O(n-1, K)$, solutions to Einstein gravity are also solutions to higher curvature gravity (2.45). In other words, the parameters $\tilde{c}_{i}^{(n)}$ do not appear in the solutions of order $O(n-1, K)$. Let us give a simple proof. Since $\mathcal{K}_{i}(\bar{R}) \sim \bar{R}^{3} \sim$ $O(n-1)^{3}$ and $O\left((n-1)^{2} K^{2}\right)$, obviously they do not contribute to the solution at order $O(n-$ $1, K)$. Now we are left with three curvature-squared terms. Notice that $\bar{R}_{\mu \nu}=0$ and $\bar{R}=0$ for all solutions to Einstein gravity with negative cosmological constant. Thus we only need to consider the GB term $\mathcal{L}_{4}(\bar{R})$, which contributes the following terms to the E.O.M

$$
P_{\mu}^{\alpha \rho \sigma} R_{\nu \alpha \rho \sigma}-\frac{1}{2} \mathcal{L}_{4}(\bar{R}) g_{\mu \nu},
$$

where $P^{\mu \alpha \rho \sigma}=\partial \mathcal{L}_{4}(\bar{R}) / \partial R_{\mu \alpha \rho \sigma}$. At leading order we have $P_{\mu}^{\alpha \rho \sigma} R_{\nu \alpha \rho \sigma} \sim 2 \bar{R}_{\mu}^{\alpha \rho \sigma} R_{\nu \alpha \rho \sigma} \sim$ $4 \bar{R}_{\mu \nu} \sim O\left((n-1)^{2}, K^{2}\right)$ and $\mathcal{L}_{4}(\bar{R}) \sim O\left((n-1)^{2}, K^{2}\right)$. Thus, it is clear that the GB term $\mathcal{L}_{4}(\bar{R})$ does not affect the E.O.M of order $O(n-1, K)$. This is indeed the case as we have seen in section 2.1. Now we finish the proof.

Finally, let us discuss the regularized boundary stress tensor of action (2.45). Let us firstly discuss the curvature-squared terms in action (2.45). Such terms are studied in [34] at the first order of $c_{2}^{(2)}$ and $c_{3}^{(2)}$. Reparameterizing their formulas, we find for the Brown-York boundary stress tensor

$$
\begin{aligned}
8 \pi \tilde{G}_{N} T_{\partial M}^{i j}= & \left(1+2 c_{1}^{(2)}(d-1)(d-2)\right)\left[K_{\partial M}^{i j}-K_{\partial M} \gamma^{i j}-(d-1) \gamma^{i j}+\frac{\Theta(d-3)}{d-2}\left(R_{\partial M}^{i j}-\frac{1}{2} R_{\partial M} \gamma^{i j}\right)\right] \\
& +2 c_{1}^{(2)}\left(Q^{i j}-\frac{1}{3} Q \gamma^{i j}\right),
\end{aligned}
$$

where $d=4$ and $Q_{i j}$ is given by eq. (2.24). Remarkably, the terms $c_{2}^{(2)} \bar{R}_{\mu \nu} \bar{R}^{\mu \nu}$ and $c_{3}^{(2)} \bar{R}^{2}$ do not contribute to the regularized boundary stress tensor. This is actually expected since for an asymptotically AdS spacetime, we can rewrite the metric in Fefferman-Graham gauge

$$
d s^{2}=g_{\mu \nu} d x^{\mu} d x^{\nu}=\frac{1}{4 \hat{\rho}^{2}} d \hat{\rho}^{2}+\frac{1}{\hat{\rho}} \gamma_{i j} d x^{i} d x^{j},
$$

\footnotetext{
${ }^{5}$ Note that we have $\bar{R}_{\mu \nu \rho \sigma}$ proportional to $O\left(K^{2}\right)$ instead of $O(K)$. The reason is as follows: $K$ depends on the orientation, while $R$ is orientation independent. Thus $R$ must be proportional to even powers of $K$. Substituting $f(r)=r^{2}-1$ and $k(r)=\sqrt{r^{2}-1} / r$ into the metric (2.28), one can check that indeed $\bar{R}_{\mu \nu \rho \sigma} \sim O\left(K^{2}\right)$.
} 
where $\gamma_{i j}=\gamma_{(0) i j}+\hat{\rho} \gamma_{(1) i j}+\cdots$ and the boundary is at $\hat{\rho} \rightarrow 0$. Near the boundary, we have $[24]$

$$
\begin{aligned}
\sqrt{g} R & \sim \sqrt{g} \sim O\left(\frac{1}{\hat{\rho^{3}}}\right), \\
\sqrt{g} \mathcal{L}_{4}(\bar{R}) & \sim O\left(\frac{1}{\hat{\rho}}\right) \\
\sqrt{g} \mathcal{K}_{7}(\bar{R}) & \sim \sqrt{g} \mathcal{K}_{8}(\bar{R}) \sim O(1), \\
\sqrt{g} \bar{R}_{\mu \nu} \bar{R}^{\mu \nu} & \sim \sqrt{g} \bar{R} \bar{R} \sim \sqrt{g} \mathcal{K}_{\mathrm{i} \neq 7,8}(\bar{R}) \sim \sqrt{g} O\left(\bar{R}^{4}\right) \sim O(\hat{\rho}) .
\end{aligned}
$$

Clearly, only terms (2.50), (2.51) in action (2.45) are divergent and need to be regularized near the boundary. No counter terms are needed for the other terms for $d=4$. In addition to the counter terms which make the action finite, one may worry about the GibbonsHawking-York (GHY) boundary terms which make a well-defined variational principle. For general higher curvature gravity, the GHY-like term is proposed in [35]. For $\mathcal{K}_{i}(\bar{R})$, we have

$$
I_{\mathrm{GHY}} \sim \int_{\partial M} d^{4} x \frac{\sqrt{\gamma}}{\hat{\rho}^{2}} P_{\hat{\rho} j}^{\hat{\rho} i} K_{\partial M}{ }_{i}^{j} \sim O(\hat{\rho}),
$$

where $P^{\mu \nu \rho \sigma}=\partial \mathcal{K}_{\mathrm{i}}(\bar{R}) / \partial R_{\mu \nu \rho \sigma}$. So the GHY-like terms for $\mathcal{K}_{i}(\bar{R})$ are harmless. The GHY-like terms and counter terms for curvature-squared are discussed in [34], which yield eq. (2.48).

In conclusion, the regularized boundary stress tensor for higher curvature gravity (2.45) is given by eq. (2.48) in dimensions less than five $(d=4)$. It should be stressed that the GHY-like terms and counter terms for $\mathcal{K}_{7}(\bar{R})$ and $\mathcal{K}_{8}(\bar{R})$ are necessary when $d \geq 6$.

\subsection{1 $f_{a}(n)$}

Applying the methods of section 2.1.1, let us calculate $f_{a}(n)$ in general higher curvature gravity (2.45). Recall that Rényi entropy on spherical entangling surface is given by

$$
S_{n}=\frac{n}{1-n} \frac{1}{T_{0}} \int_{T_{0} / n}^{T_{0}} S_{\mathrm{BH}}(T) d T
$$

with $S_{\mathrm{BH}}(T)$ the black hole entropy

$$
S_{\mathrm{BH}}=\frac{1}{8 G_{N}} \int_{H} d y^{3} \sqrt{h} \frac{\partial L}{\partial R_{\mu \nu \rho \sigma}} \varepsilon_{\mu \nu} \varepsilon_{\rho \sigma} .
$$

To suppress the massive modes and ghost modes with $M \sim 1 / c_{\mathrm{i}}^{(n)}$, we work in perturbative framework with $c_{\mathrm{i}}^{(n)} \ll 1$. After some calculations, we find the black hole solution as

$$
d s_{\text {bulk }}^{2}=\frac{d r^{2}}{f(r)}+f(r) F(r) d \tau^{2}+r^{2} d \Sigma_{3}^{2}
$$

where $d \Sigma_{3}^{2}$ is the line element for hyperbolic plane $H^{3}$ with unit curvature, and $f(r), F(r)$ are given by 


$$
\begin{aligned}
f(r)= & r^{2}-1+\frac{2(n-1)}{3 r^{2}} \\
& -\frac{\left(r^{6}\left(336 c_{1}^{(2)}+192 c_{7}^{(3)}-96 c_{8}^{(3)}+35\right)-24 r^{2}\left(c_{1}^{(2)}+228 c_{7}^{(3)}-3 c_{8}^{(3)}\right)+4608 c_{7}^{(3)}\right)}{27\left(1+8 c_{1}^{(2)}\right) r^{8}}(n-1)^{2}, \\
& +O(n-1)^{3} \\
F(r)= & 1-\frac{8\left(52 c_{7}^{(3)}+3 c_{8}^{(3)}\right)}{3\left(1+8 c_{1}^{(2)}\right) r^{8}}(n-1)^{2}+O(n-1)^{3} .
\end{aligned}
$$

From the conditions

$$
\begin{aligned}
f\left(r_{H}\right) & =0, \\
T & =\left.\frac{1}{4 \pi} \sqrt{f^{\prime}(r) \partial_{r}[f(r) F(r)]}\right|_{r=r_{H}}=\frac{1}{2 \pi n},
\end{aligned}
$$

we find a consistent solution

$$
r_{H}=1-\frac{(n-1)}{3}+\frac{10}{27}(n-1)^{2}+\frac{4\left(4 c_{1}^{(2)}-84 c_{7}^{(3)}-3 c_{8}^{(3)}\right)}{27\left(1+8 c_{1}^{(2)}\right)}(n-1)^{2}+O(n-1)^{3} .
$$

Substituting the above equations into eqs. (2.55), (2.56), we obtain

$$
\frac{8 G_{N}}{\pi} f_{a}(n)=1-\frac{1+8 c_{1}^{(2)}}{2}(n-1)+\frac{1}{54}\left(336 c_{1}^{(2)}+192 c_{7}^{(3)}-96 c_{8}^{(3)}+35\right)(n-1)^{2}+\cdots
$$

Using the following relations [32],

$$
\begin{aligned}
a & =\frac{\pi}{8 G_{N}}, \quad c=\frac{\pi}{8 G_{N}}\left(1+8 c_{1}^{(2)}\right), \\
t_{2} & =\frac{12}{1+8 c_{1}^{(2)}}\left(4 c_{1}^{(2)}-192 c_{7}^{(3)}+96 c_{8}^{(3)}\right), \\
t_{4} & =\frac{2160}{1+8 c_{1}^{(2)}}\left(2 c_{7}^{(3)}-c_{8}^{(3)}\right),
\end{aligned}
$$

we can rewrite eq. (2.63) as

$$
f_{a}(n)=a-\frac{c}{2}(n-1)+c\left(\frac{35}{54}+\frac{7}{324} t_{2}+\frac{1}{84} t_{4}\right)(n-1)^{2}+\cdots
$$

which is eq. (1.9) advertised in the Introduction.

We remark that although we work in linear order of $c_{\mathrm{i}}^{(n)}$ in the above derivation, our result eq. (2.67) applies to finite $c_{\mathrm{i}}^{(n)}$. For the case $c_{2}^{(2)}=c_{3}^{(2)}=0$, eqs. (2.58), (2.59), (2.62) are exact in $c_{\mathrm{i}}^{(n)}$. For small but non-zero $c_{2}^{(2)}$ and $c_{3}^{(2)}$, we have performed a fifth order perturbation and find that eq. (2.67) remains unchanged. 


\subsection{2 $f_{c}(n)$}

Now let us study $f_{c}(n)$ in higher curvature gravity (2.45). Similarly, we consider the first order variation (2.17) of the partition function with $T_{i j}$ computed on the undeformed hyperboloid background. Here $T^{i j}$ is the regularized boundary stress tensor given by eq. (2.48). The bulk metric takes the form

$$
d s_{\text {bulk }}^{2}=\frac{d r^{2}}{f(r)}+f(r) F(r) d \tau^{2}+\frac{r^{2}}{\rho^{2}}\left[d \rho^{2}+\left(\delta_{\hat{i} \hat{j}}+q(r) Q_{a b i \hat{j}} x^{a} x^{b}+O\left(\rho^{3}\right)\right) d y^{\hat{i}} d y^{\hat{j}}\right],
$$

which approaches the deformed hyperboloid eq. (2.19) for $q(\infty)=1$. Recalling $f(r), F(r)$ as given in eqs. (2.58), (2.59) and substituting all these equations together with $\gamma_{\hat{i} \hat{j}}=$ $\frac{r^{2}}{\rho^{2}} Q_{a b \hat{i} \hat{j}} x^{a} x^{b}$ into eqs. (2.17), (2.18), we obtain

$$
\frac{8 G_{N}}{\pi} f_{c}(n)=\left(1+8 c_{1}^{(2)}\right)+\left(-\frac{17}{18}-\frac{32}{3} c_{1}^{(2)}-\frac{32}{3} c_{7}^{(3)}+\frac{16}{3} c_{8}^{(3)}\right)(n-1)+\cdots
$$

Applying eqs. (2.64), (2.65), (2.66), we can rewrite $f_{c}(n)$ as

$$
f_{c}(n)=c+c\left(-\frac{17}{18}-\frac{7}{108} t_{2}-\frac{1}{27} t_{4}\right)(n-1)+\cdots .
$$

Notice that $f_{a}(n)(2.67)$ and $f_{c}(n)(2.70)$ are consistent with identity (1.7). This is a non-trivial check of our holographic approach, in particular, the regularized boundary stress tensor eq. (2.48).

\subsection{3 $f_{b}(n)$}

Finally, let us discuss $f_{b}(n)$ in the higher derivative gravity. Similar to the case of the GB gravity, the key point is to find deformed black hole solutions up to order $O(K)$

$$
d s_{\text {bulk }}^{2}=\frac{d r^{2}}{f(r)}+f(r) F(r) d \tau^{2}+\frac{r^{2}}{\rho^{2}}\left[d \rho^{2}+\left(\delta_{\hat{i} \hat{j}}+k(r) K_{a \hat{i} \hat{j}} x^{a}+O\left(\rho^{2}\right)\right) d y^{i} d y^{j}\right]
$$

For traceless $K_{a i j}$, there is one independent equation of $k(r)$. We find the solution at the linear order in $(n-1)$ is exactly the same as that of Einstein gravity, which agrees with the arguments below eq. (2.46). Modifications from the higher-curvature terms only appear at higher orders. Remarkably, at the next order $O(n-1)^{2}$, only $c_{1}^{(2)}, c_{7}^{(3)}$ and $c_{8}^{(3)}$ contribute.

Following the approach of section 2.1.3, we obtain $k(r)$ in large $r$ expansion as

$$
k(r)=1-\frac{1}{2 r^{2}}+\frac{\beta_{n}}{r^{4}}+O\left(\frac{1}{r^{6}}\right)
$$

where

$$
\beta_{n}=-\frac{1}{8}+\frac{n-1}{12}+\frac{\left(-600 c_{1}^{(2)}+4224 c_{7}^{(3)}-2112 c_{8}^{(3)}-67\right)}{432\left(1+8 c_{1}^{(2)}\right)}(n-1)^{2}+O(n-1)^{3} .
$$


Substituting eqs. (2.72), (2.58), (2.59) and $\delta \gamma_{\hat{i} \hat{j}}=\frac{r^{2}}{\rho^{2}} K_{a \hat{i} \hat{j}} x^{a}$ into (2.17) and (2.26), we obtain

$$
\begin{aligned}
f_{b}(n) & =\frac{\pi}{8 G_{N}}\left[\left(1+8 c_{1}^{(2)}\right)+\left(-\frac{11}{12}-10 c_{1}^{(2)}+32 c_{7}^{(3)}-16 c_{8}^{(3)}\right)(n-1)+\cdots\right] \\
& =c-c\left(\frac{11}{12}+\frac{1}{18} t_{2}+\frac{1}{45} t_{4}\right)(n-1)+\cdots
\end{aligned}
$$

as declared in the Introduction.

Now we have obtained $f_{a}^{\prime \prime}(1), f_{b}^{\prime}(1)$ and $f_{c}^{\prime}(1)$ by using holographic methods. Interestingly, they only depend on the parameters of stress tensor two-point and three-point functions. When $c_{2}^{(2)}=c_{3}^{(2)}=0$, our derivations are nonperturbative in the coupling constants of higher curvature gravity. For small but non-zero $c_{2}^{(2)}$ and $c_{3}^{(2)}$, we have performed a fifth order perturbation and find that they remains unchanged. In conclusion, our obtained results eqs. (1.9), (1.10), (1.11) are universal laws for strongly coupled CFTs that are dual to general higher curvature gravity. It is expected that there are no such universal laws at the next order, since the next order terms would involve the stress energy four-point functions which no longer admit any universal form.

\section{The story of free CFTs}

In this section, we discuss the universal terms of Rényi entropy for free CFTs. We find the holographic relations found in section2 also apply to free fermions and free vectors but not to free scalars. We find a combined relation which is obeyed by all free CFTs and strongly coupled CFTs with holographic dual. It seems that this combined relation is universal for all CFTs in four dimensions.

For the theory consisting of $n_{s}$ free real scalars, $n_{f}$ free Weyl fermions and $n_{v}$ free vectors, the functions $f_{a}(n)$ and $f_{c}(n)$ have been calculated explicitly in $[8,15,36-39]$. We list the results as follows:

$$
\begin{aligned}
& f_{a}(n)=\frac{1}{360}\left[n_{s} \frac{(1+n)\left(1+n^{2}\right)}{4 n^{3}}+n_{f} \frac{(1+n)\left(7+37 n^{2}\right)}{16 n^{3}}+n_{v} \frac{\left(1+n+31 n^{2}+91 n^{3}\right)}{2 n^{3}}\right] \\
& f_{c}(n)=\frac{1}{120}\left[n_{s} \frac{(1+n)\left(1+n^{2}\right)}{4 n^{3}}+n_{f} \frac{(1+n)\left(7+17 n^{2}\right)}{16 n^{3}}+n_{v} \frac{\left(1+n+11 n^{2}+11 n^{3}\right)}{2 n^{3}}\right]
\end{aligned}
$$

One can check that the above $f_{a}(n)$ and $f_{c}(n)$ satisfy the identity eq. (1.7). Assuming $f_{b}(n)=f_{c}(n)$, we have

$$
f_{b}(n)=\frac{1}{120}\left[n_{s} \frac{(1+n)\left(1+n^{2}\right)}{4 n^{3}}+n_{f} \frac{(1+n)\left(7+17 n^{2}\right)}{16 n^{3}}+n_{v} \frac{\left(1+n+11 n^{2}+11 n^{3}\right)}{2 n^{3}}\right] .
$$

This is at least the case for free scalars [12]. Numerical calculations also support $f_{b}(n)=$ $f_{c}(n)$ for free fermions [17]. 
According to [40, 41], the stress tensor three-point functions for CFTs in general spacetime dimensions are completely determined in terms of the three parameters $A, B, C$ as,

$$
\begin{aligned}
C_{T} & =\frac{\pi^{d / 2}}{d(d+2) \Gamma[d / 2]}[(d-1)(d+2) A-2 B-4(d+1) C], \\
t_{2} & =\frac{2(d+1)}{d} \frac{(d-2)(d+2)(d+1) A+3 d^{2} B-4 d(2 d+1) C}{(d-1)(d+2) A-2 B-4(d+1) C}, \\
t_{4} & =-\frac{(d+1)}{d} \frac{(d+2)\left(2 d^{2}-3 d-3\right) A+2 d^{2}(d+2) B-4 d(d+1)(d+2) C}{(d-1)(d+2) A-2 B-4(d+1) C},
\end{aligned}
$$

where for free $4 \mathrm{~d}$ CFTs, we have

$$
\begin{aligned}
A & =\frac{8}{27 \pi^{6}}\left(n_{s}-54 n_{v}\right) \\
B & =-\frac{2}{27 \pi^{6}}\left(8 n_{s}+432 n_{v}+27 n_{f}\right) \\
C & =-\frac{1}{27 \pi^{6}}\left(2 n_{s}+432 n_{v}+27 n_{f}\right) .
\end{aligned}
$$

and $C_{T}=\frac{40}{\pi^{4}} c$.

Substituting eqs. (3.7)-(3.6) into the holographic relations eqs. (1.9), (1.10), (1.11) for $f_{a}, f_{b}, f_{c}$ and comparing with those of free CFTs eqs. (3.1), (3.2), (3.3), we find exact agreements for fermions and vectors. However, there is discrepancy for scalars. As noticed in [20], such discrepancy results from the boundary contributions to the modular Hamiltonian. Interestingly, we find the following combined holographic relations

$$
\begin{aligned}
2 f_{b}^{\prime}(1)-3 f_{c}^{\prime}(1) & =c\left(1+\frac{1}{12} t_{2}+\frac{1}{15} t_{4}\right) \\
2 f_{b}^{\prime}(1)+\frac{9}{2} f_{a}^{\prime \prime}(1) & =c\left(4+\frac{1}{12} t_{2}+\frac{1}{15} t_{4}\right)
\end{aligned}
$$

are satisfied by all free CFTs including scalars. We conjecture these are universal laws for all CFTs in four dimensions. As mentioned in the Introduction, eq. (3.10) and eq. (3.11) are not independent, which can be derived from each other by applying eq. (1.7).

In the notation of [12], our conjecture (3.10) becomes

$$
\pi C_{D}^{\prime \prime}(1)-36 h_{n}^{\prime \prime}(1)=\frac{2 \pi^{3}}{5} C_{T}\left(1+\frac{1}{12} t_{2}+\frac{1}{15} t_{4}\right), \quad(\text { for } 4 \mathrm{~d} \mathrm{CFTs}),
$$

where $C_{T}=\frac{40}{\pi^{4}} c$ for $4 \mathrm{~d}$. As the quantities $h_{n}$ and $C_{D}$ have natural definitions in all dimensions. It is expected that one can generalize our results to general dimensions. We will perform this analysis in the next section.

\section{Universality of HRE in general dimensions}

In this section, we study $h_{n}(n)$ and $C_{D}(n)$ of holographic Rényi entropy for CFT in general $d$-dimensions. We firstly consider the $3 \mathrm{~d}$ case and then discuss the case in higher dimensions. We find that in general dimensions there are indeed similar holographic universal 
laws expressing $h_{n}^{\prime \prime}(1)$ and $C_{D}^{\prime \prime}(1)$ in terms of a linear combination of $C_{T}, t_{2}$ and $t_{4}$. And for all the examples we have checked, these holographic laws are obeyed by free fermions, but are violated by free scalars. Similar to what we did above for four dimensions, we are also able to find a specific relation involving linearly the quantities $h_{n}^{\prime \prime}(1), C_{D}^{\prime \prime}(1), C_{T}, t_{2}$ and $t_{4}$, which applies to free fermions, free scalars and strongly coupled CFTs with holographic dual. We conjecture that this relation holds for general CFTs.

To proceed, we apply the holographic approach developed in $[10,13]$ to derive $h_{n}(n)$ and $C_{D}(n)$ for general higher curvature gravity. This procedure treats the extrinsic curvature perturbatively. For our purpose, we only need to consider the linear order of the extrinsic curvature below. Inspired by [10, 13], we consider the following bulk metric

$$
\begin{aligned}
d s_{\text {bulk }}^{2}= & \frac{d r^{2}}{f(r)}+f(r) F(r) d \tau^{2} \\
& +\frac{r^{2}}{\rho^{2}}\left[d \rho^{2}+\left(\delta_{\hat{i} \hat{j}}+2 k(r) \bar{K}_{a \hat{i} \hat{j}} x^{a}\right) d y^{\hat{i}} d y^{\hat{j}}+\frac{4}{d-2} k(r) \partial_{i} K^{a} x_{a} \rho d \rho d y^{\hat{i}}+O\left(\rho^{2}\right)\right],
\end{aligned}
$$

where $\bar{K}_{a \hat{i} \hat{j}}$ is the traceless part of extrinsic curvature and we have $\partial_{\hat{k}} K_{a \hat{i} \hat{j}}=\partial_{\hat{j}} K_{a \hat{i} \hat{k}}+O\left(K^{2}\right)$ for consistency [10]. According to $[10,13,42], h_{n}$ and $C_{D}(n)$ can be extracted from the boundary stress tensor

$$
\begin{aligned}
\left\langle T_{a b}(x)\right\rangle_{n} & =\frac{g_{n}}{\rho^{2}}\left((d-1) \delta_{a b}-d \frac{x_{a} x_{b}}{\rho^{2}}\right)+\cdots, \\
\left\langle T_{a \hat{i}}(x)\right\rangle_{n} & =\frac{x_{a} x_{b}}{\rho^{2}} \partial_{\hat{i}} K^{b} \frac{k_{n}}{d-2}+\cdots, \\
\left\langle T_{\hat{i} \hat{j}}(x)\right\rangle_{n} & =\frac{1}{\rho^{2}}\left(-g_{n} \delta_{\hat{i} \hat{j}}+k_{n} \bar{K}_{\hat{i} \hat{j}}^{a} x_{a}\right)+\cdots,
\end{aligned}
$$

where

$$
k_{n}-k_{1}=\frac{(d-1) \Gamma\left(\frac{d}{2}-1\right) \pi^{\frac{d}{2}-2}}{2 \Gamma(d+1)} \frac{C_{D}}{n}-\frac{3 d-4}{d-2} \frac{h_{n}}{2 \pi n}, \quad \text { and } \quad g_{n}-g_{1}=\frac{h_{n}}{2 \pi n} .
$$

The boundary stress tensor in general higher curvature gravity has been calculated in [32], yielding that

$$
\left\langle T_{i j}\right\rangle=\frac{d}{f_{d}} C_{T} h_{i j}^{(d)}=\frac{d}{16 \pi G_{N}}\left(1+4(d-2) c_{1}^{(2)}\right) h_{i j}^{(d)},
$$

where

$$
\begin{aligned}
f_{d} & =2 \frac{d+1}{d-1} \frac{\Gamma(d+1)}{\pi^{d / 2} \Gamma(d / 2)}, \\
C_{T} & =\frac{f_{d}}{16 \pi G_{N}}\left(1+4(d-2) c_{1}^{(2)}\right) .
\end{aligned}
$$

and $h_{i j}^{(d)}$ appears in the Fefferman-Graham expansion of the asymptotic AdS metric

$$
d s^{2}=\frac{d z^{2}}{z^{2}}+\frac{1}{z^{2}}\left(g_{i j}^{(0)}+z^{2} g_{i j}^{(1)}+\cdots+z^{d} h_{i j}^{(d)}+\cdots\right) d y^{i} d y^{j}
$$


Notice that the stress-tensor eq. (4.4) contains contributions from the $g_{(0) i j}$ in even dimensions [26]. These contributions reflect the presence of conformal anomalies. However, as argued in [10], these terms do not affect $C_{D}(n)$ and $h_{n} \cdot{ }^{6}$ So we have ignored them in the present paper. Note also that we use a seemingly different stress tensor $T_{\partial M i j}$ eq. (2.48) in section 2. Actually, the stress-tensor eq. (2.48) is equivalent to eq. (4.4) up to a rescaling and some functions of $g_{(0) i j}[26]$

$$
\left\langle T_{i j}\right\rangle=\lim _{z \rightarrow 0} \frac{1}{z^{d-2}} T_{\partial M i j}
$$

If we take the stress tensor eq. (4.4) instead of eq. (2.48) in the procedure of section 2, we get the same results for $f_{b}(n)$ and $f_{c}(n)$. The interested reader is referred to appendix A for the proof of the equivalence. Now let us focus on the stress tensor eq. (4.4) from now on.

Comparing eq. (4.4) with eq. (4.2), one can read out $h_{n}(n)$ and $C_{D}(n)$. Let us take Einstein gravity as an example. The solution is given by

$$
\begin{aligned}
& f(r)=r^{2}-1-\frac{M}{r^{d-2}}, \quad F(r)=1, \\
& k(r)=\frac{\sqrt{r^{2}-1}}{r}+\frac{\beta_{n}}{r^{d}}+O\left(\frac{1}{r^{d+1}}\right) .
\end{aligned}
$$

From the above equations, one can easily obtain

$$
h_{\hat{i} \hat{j}}^{(d)}=\frac{1}{\rho^{2}}\left[\left(\frac{1}{d} M+g_{0}\right) \delta_{\hat{i} \hat{j}}+\left(\frac{2}{d} M+2 \beta_{n}+k_{0}\right) \bar{K}_{\hat{i} \hat{j}}^{a} x_{a}\right],
$$

where $g_{0}$ and $k_{0}$ are constants which are not important. ${ }^{7}$ Comparing eqs. (4.4), (4.11) with the last equation of (4.2), one obtains $[10,13]$

$$
\begin{aligned}
\frac{h_{n}}{n} & =-\frac{M}{8 G_{N}}, \\
\frac{C_{D}}{n} & =\frac{d \Gamma(d+1)}{(d-1) \pi^{d / 2-2} \Gamma(d / 2)} \frac{2(d-2)\left(\beta_{n}-\beta_{1}\right)-M}{16 \pi G_{N}} .
\end{aligned}
$$

Now let us turn to discuss the general higher curvature gravity (2.45). In general, it is difficult to find the black hole solutions for higher derivative gravity. For simplicity, we work in the perturbative framework of the coupling constants $c_{i}^{(n)}$. Remarkably, we find the solutions behaving as

$$
\begin{aligned}
& f(r)=r^{2}-1-\frac{M_{e}}{r^{d-2}}+O\left(\frac{1}{r^{d}}\right), \quad F(r)=1+O\left(\frac{1}{r^{2 d}}\right), \\
& k(r)=\frac{\sqrt{r^{2}-1}}{r}+\frac{\beta_{n}}{r^{d}}+O\left(\frac{1}{r^{d+1}}\right) .
\end{aligned}
$$

\footnotetext{
${ }^{6}$ One can easily check that $g_{(0) i j}$ is independent of $M_{e}$ and $\beta_{n}$. Thus, the contributions to the stresstensor eq. (4.4) from $g_{(0) i j}$ do not affect $C_{D}(n)$ and $\beta_{n}$.

${ }^{7}$ From eqs. (4.2), (4.4), (4.11), we can derive $k_{n}$ and $g_{n}$, which have a linear dependence on the constants $g_{0}$ and $k_{0}$ appearing in eq. (4.11). However, we are interested of $C_{D}(n)$ and $h_{n}$ instead of $k_{n}$ and $g_{n}$. Since $C_{D}(n)$ and $h_{n}$ are functions of $\left(k_{n}-k_{1}\right)$ and $\left(g_{n}-g_{1}\right)$ from eq. (4.3). They do not depend on $g_{0}$ and $k_{0}$ instead.
} 
Here 'e' denotes effective. Using the above solutions, we can work out $h_{\hat{i} \hat{j}}^{(d)}$ in the FeffermanGraham expansion. Interestingly, it takes exactly the same form as that of Einstein gravity eq. (4.11), only replacing $M$ and $\beta_{n}$ by the effective counterparts $M_{e}$ and $\beta_{n}$. Comparing eqs. (4.4) with eq. (4.2), we finally obtain

$$
\begin{aligned}
& \frac{h_{n}}{C_{T}}=-2 \pi n \frac{M_{e}}{f_{d}} \\
& \frac{C_{D}}{C_{T}}=\frac{d \pi^{2} n}{d+1}\left[(d-2)\left(\beta_{n}-\beta_{1}\right)-\frac{M_{e}}{2}\right]
\end{aligned}
$$

where $f_{d}$ and $C_{T}$ are given by (4.5) and (4.6). $h_{n}$ and $C_{D}$ were first obtained for Einstein Gravity in [13] and for Gauss-Bonnet Gravity in [10]. Here we derive them for the general higher curvature gravity. It is remarkable that, when expressed in terms of $M_{e}$ and the $\beta_{n}$ 's, the coefficients $h_{n}$ and $C_{D}$ take on these very simple universal forms (4.16), (4.17). As a first check, our formulae agree with those of $[10,13]$ for Einstein gravity and GaussBonnet Gravity. The holographic relations (4.16) and (4.17) are one of the main results we obtain for general dimensional CFTs. It should be mentioned that $G_{N}$ and $c_{i}^{(n)}$ appearing in this section are actually $\tilde{G}_{N}$ and $\tilde{c}_{\mathrm{i}}^{(n)}$ defined in the action $(2.45)$. For simplicity, we have ignored the notation .

\subsection{CFTs in three dimensions}

In this section, we use the formulas obtained in the above section to study the universal behaves of $h_{n}^{\prime \prime}(1)$ and $C_{D}^{\prime \prime}(1)$ for 3d CFTs. We need to solve the E.O.M of general higher curvature to get the effective mass $M_{e}$ and $\beta_{n}$. Note that the Gauss-Bonnet term is a total derivative in four-dimensional spacetime. Without loss of generality, we can set $c_{1}^{(2)}=0$. After some calculations, we derive

$$
\begin{aligned}
f(r)= & r^{2}-1+\frac{n-1}{r}-\frac{3\left(r^{5}\left(8 c_{7}^{(3)}-4 c_{8}^{(3)}+5\right)+4 r^{2}\left(c_{8}^{(3)}-44 c_{7}^{(3)}\right)+144 c_{7}^{(3)}\right)(n-1)^{2}}{8 r^{6}} \\
& +O(n-1)^{3}, \\
F(r)= & 1-\frac{9\left(12 c_{7}^{(3)}+c_{8}^{(3)}\right)}{2 r^{6}}(n-1)^{2}+O(n-1)^{2} .
\end{aligned}
$$

One can see that these solutions obey the behaving (4.14) and the effective mass is given by

$$
M_{e}=-(n-1)+\frac{3\left(8 c_{7}^{(3)}-4 c_{8}^{(3)}+5\right)}{8}(n-1)^{2}+O(n-1)^{2} .
$$

Note that we have used the conditions $f\left(r_{H}\right)=F\left(r_{H}\right)=0$ and $T=\frac{1}{2 \pi n}$ to fix the constants of integration for $f(r)$ and $F(r)$, with $r_{H}$ given by

$$
r_{H}=1-\frac{n-1}{2}+\frac{9}{16}(n-1)^{2}-\frac{9 c_{7}^{(3)}(n-1)^{2}}{2}+O(n-1)^{3} .
$$


Solving $k(r)$ up to order $O(n-1)^{2}$, we obtain

$$
\begin{aligned}
k(r) & =\frac{\sqrt{r^{2}-1}}{r}+\frac{\beta_{n}}{r^{3}}+O\left(\frac{1}{r^{4}}\right) \\
\beta_{n} & =\frac{n-1}{6}+\left(\frac{19 c_{7}^{(3)}}{2}-\frac{19 c_{8}^{(3)}}{4}-\frac{41}{144}\right)(n-1)^{2}+O(n-1)^{3}
\end{aligned}
$$

Substituting eqs. (4.20), (4.23) into eqs. (4.16), (4.17), we obtain

$$
\begin{aligned}
& \frac{h_{n}}{C_{T}}=\frac{1}{24} \pi^{3}(n-1)-\frac{\pi^{3}}{11520}\left(420+t_{4}\right)(n-1)^{2}+O(n-1)^{3}, \\
& \frac{C_{D}}{C_{T}}=\frac{1}{2} \pi^{2}(n-1)-\frac{\pi^{2}}{240}\left(100-t_{4}\right)(n-1)^{2}+O(n-1)^{3} .
\end{aligned}
$$

where we have used [32] $t_{4}=720\left(2 c_{7}^{(3)}-c_{8}^{(3)}\right)$.

Now let us compare our holographic results with those of free CFTs. $h_{n}$ for free fermions and free scalars are calculated in [20,36, 43-45]. And it is proved in [43, 44] that $C_{D}=d \Gamma\left(\frac{d+1}{2}\right)\left(\frac{2}{\sqrt{\pi}}\right)^{d-1} h_{n}$ for free fermions and scalars in three dimensions. For free Dirac fermions, we have [45]

$$
C_{T}=\frac{3}{16 \pi^{2}}, t_{4}=-4, h_{n}^{\prime}(1)=\frac{\pi}{128}, h_{n}^{\prime \prime}(1)=-\frac{13 \pi}{960}, C_{D}^{\prime}(1)=\frac{3}{32}, C_{D}^{\prime \prime}(1)=-\frac{13}{80},
$$

which exactly match the holographic results eqs. (4.24), (4.25). However, similar to the case of $4 \mathrm{~d}$ CFTs, mismatch appears for free scalars. According to [20, 43], it is

$$
C_{T}=\frac{3}{16 \pi^{2}}, t_{4}=4, h_{n}^{\prime}(1)=\frac{\pi}{128}, h_{n}^{\prime \prime}(1)=-\frac{17 \pi}{960}, C_{D}^{\prime}(1)=\frac{3}{32}, C_{D}^{\prime \prime}(1)=-\frac{17}{80},
$$

for free complex scalars. It is found in $[20,45]$ there is discrepancy for $h_{n}^{\prime \prime}(1)$. Here we note further that there is a discrepancy in $C_{D}^{\prime \prime}(1)$ too. Similar to the $4 \mathrm{~d}$ case, we find a combination of $h_{n}^{\prime \prime}(1)$ and $C_{D}^{\prime \prime}(1)$,

$$
\pi C_{D}^{\prime \prime}(1)-16 h_{n}^{\prime \prime}(1)=\frac{\pi^{3}}{3} C_{T}\left(1+\frac{t_{4}}{30}\right)
$$

which is obeyed by free scalars, free fermions and CFTs with gravity dual. In addition to free CFTs and strongly coupled CFTs with gravity dual, it is interesting to investigate whether the 'universal law' (4.28) is obeyed by more general CFTs.

\subsection{CFTs in higher dimensions}

Let us go on to discuss $h_{n}$ and $C_{D}$ in higher dimensions. Similar to the cases of $3 \mathrm{~d}$ CFTs and $4 \mathrm{~d}$ CFTs, we need to solve the E.O.M in the bulk to get the effective mass and $\beta_{n}$. Then we can derive $h_{n}$ and $C_{D}$ from the general formula eqs. (4.16), (4.17). 
By solving the E.O.M for the general higher curvature gravity (2.45), we obtain

$$
\begin{aligned}
f(r) & =r^{2}-1-\frac{M_{\operatorname{Ein}}}{r^{d-2}}+\frac{c_{1}^{(2)} f_{1}(r)+c_{7}^{(3)} f_{7}(r)+c_{8}^{(3)} f_{8}(r)}{1+4(d-2) c_{1}^{(2)}}(n-1)^{2}+O(n-1)^{3} \\
& =r^{2}-1-\frac{M_{e}}{r^{d-2}}+O\left(\frac{1}{r^{d}}\right) \\
F(r) & =1+\frac{c_{7}^{(3)} F_{7}(r)+c_{8}^{(3)} F_{8}(r)}{1+4(d-2) c_{1}^{(2)}}(n-1)^{2}+O(n-1)^{3}=1+O\left(\frac{1}{r^{2 d}}\right) \\
k(r) & =\frac{\sqrt{r^{2}-1}}{r}+\frac{\beta_{n}}{r^{d}}+O\left(\frac{1}{r^{d+1}}\right)
\end{aligned}
$$

where

$$
\begin{aligned}
M_{e}= & -\frac{2}{d-1}(n-1)+\frac{(2 d-3)(2 d-1)}{(d-1)^{3}}(n-1)^{2} \\
& +\frac{c_{1}^{(2)} m_{1}+c_{7}^{(3)} m_{7}+c_{8}^{(3)} m_{8}}{1+4(d-2) c_{1}^{(2)}}(n-1)^{2}+O(n-1)^{3},
\end{aligned}
$$

and

$$
\begin{aligned}
\beta_{n}= & \beta_{1}+\frac{1}{d(d-1)}(n-1)-\frac{4 d^{3}-8 d^{2}+d+2}{2 d^{2}(d-1)^{3}}(n-1)^{2} \\
& +\frac{c_{1}^{(2)} b_{1}+c_{7}^{(3)} b_{7}+c_{8}^{(3)} b_{8}}{1+4(d-2) c_{1}^{(2)}}(n-1)^{2}+O(n-1)^{3} .
\end{aligned}
$$

Here $f_{1}(r), f_{7}(r), f_{8}(r), F_{7}(r), F_{8}(r), m_{1}, m_{2}, m_{3}, b_{1}, b_{7}, b_{8}$ are determined by the E.O.M. We have worked out the solutions case by case up to $d=9$. Please refer to the appendix for these solutions. In summary we obtain:

$$
\begin{aligned}
& \frac{h_{n}}{C_{T}}=2 \pi^{\frac{d}{2}+1} \frac{\Gamma\left(\frac{d}{2}\right)}{\Gamma(d+2)}(n-1)+\frac{h_{n}^{\prime \prime}(1)}{2 C_{T}}(n-1)^{2}+O(n-1)^{3}, \\
& \frac{C_{D}}{C_{T}}=\frac{2 \pi^{2}}{d+1}(n-1)+\frac{C_{D}^{\prime \prime}(1)}{2 C_{T}}(n-1)^{2}+O(n-1)^{3},
\end{aligned}
$$

with $\frac{h_{n}^{\prime \prime}(1)}{C_{T}}$ and $\frac{C_{D}^{\prime \prime}(1)}{C_{T}}$ given by

$$
\begin{aligned}
\frac{h_{n}^{\prime \prime}(1)}{C_{T}}= & -\frac{2 \pi^{\frac{d}{2}+1} \Gamma\left(\frac{d}{2}\right)}{(d-1)^{3} d(d+1) \Gamma(d+3)}\left[d\left(2 d^{5}-9 d^{3}+2 d^{2}+7 d-2\right)\right. \\
& \left.+(d-2)(d-3)(d+1)(d+2)(2 d-1) t_{2}+(d-2)\left(7 d^{3}-19 d^{2}-8 d+8\right) t_{4}\right], \\
\frac{C_{D}^{\prime \prime}(1)}{C_{T}}= & \frac{4 \pi^{2}}{d+1}\left[\frac{1-d^{2}+d}{d^{2}-d}-\frac{(d-2)(d-3)}{(d-1)^{2} d} t_{2}-\frac{(d-2)\left(3 d^{2}-7 d-8\right)}{(d-1)^{2} d(d+1)(d+2)} t_{4}\right] .
\end{aligned}
$$

Note that the coefficients of $t_{2}$ and $t_{4}\left(t_{2}\right)$ in $h_{n}^{\prime \prime}(1)$ and $C_{D}^{\prime \prime}(1)(4.36),(4.37)$ vanish when $d=2(d=3)$. This is the expected result, which can be regarded as a check of our general formula (4.36), (4.37). One can also check that the general formulas (4.36), (4.37) reproduce 
the results of $3 \mathrm{~d}$ and $4 \mathrm{~d}$ CFTs. We remark that the holographic formula of $h_{n}^{\prime \prime}(1)(4.36)$ agrees with the those of $[20,45]$, which are derived by using three-point functions of stress tensor. As they have checked, the relation (4.36) for $h_{n}^{\prime \prime}(1)$ works well for free fermions (up to $d=12)$ but not for free scalars $(d>2)$.

Before we end this section, let us make some comments about the possible universal relation between $C_{D}$ and $h_{n}$. For general dimensions, the generalization of the $4 \mathrm{~d}$ conjecture (1.8) is the statement [12]:

$$
C_{D}(n)=d \Gamma\left(\frac{d+1}{2}\right)\left(\frac{2}{\sqrt{\pi}}\right)^{d-1} h_{n}(n) .
$$

This relation can be motivated by the observation that if one assume (4.38) holds for free fermions and conformal tensor fields, one can prove $C_{D}^{\prime \prime}(1)$ of these fields exactly match the holographic formula (4.37). Turning the logic around, if one assume free fermions and conformal tensor fields obey the holographic formulas $(4.36),(4.37),{ }^{8}$ one can prove that the weaker relation

$$
C_{D}^{\prime \prime}(1)=d \Gamma\left(\frac{d+1}{2}\right)\left(\frac{2}{\sqrt{\pi}}\right)^{d-1} h_{n}^{\prime \prime}(1)
$$

holds in general dimensions. In proving these, we have found useful the relations (4.6), (3.5), (3.6) and that

$$
\begin{aligned}
& A=\frac{1}{S_{d}^{3}}\left[\frac{d^{3}}{(d-1)^{3}} n_{s}-\frac{d^{3}}{d-3} \tilde{n}_{t}\right], \\
& B=-\frac{1}{S_{d}^{3}}\left[\frac{(d-2) d^{3}}{(d-1)^{3}} n_{s}+\frac{d^{2}}{2} \tilde{n}_{f}+\frac{(d-2) d^{3}}{d-3} \tilde{n}_{t}\right], \\
& C=-\frac{1}{S_{d}^{3}}\left[\frac{(d-2)^{2} d^{2}}{4(d-1)^{3}} n_{s}+\frac{d^{2}}{4} \tilde{n}_{f}+\frac{(d-2) d^{3}}{2(d-3)} \tilde{n}_{t}\right],
\end{aligned}
$$

where $S_{d}=2 \pi^{d / 2} / \Gamma\left(\frac{d}{2}\right), \tilde{n}_{f}=\operatorname{tr}(1) n_{f}=2^{[d / 2]} n_{f}, n_{f}$ is the number of Dirac fermion, tr is the Dirac trace and $\tilde{n}_{t}$ denotes the number of degrees of freedom contributed by the $(n-1)$-form in even dimensions $d=2 n$ [19]. However incompatiblity arises in the scalar sector as before. Indeed using (4.6), (3.5), (3.6) and (4.40)-(4.42) in the holographic formulas (4.36), (4.37), we find for a free theory with $n_{s}$ scalars,

$$
C_{D}^{\prime \prime}(1)-d \Gamma\left(\frac{d+1}{2}\right)\left(\frac{2}{\sqrt{\pi}}\right)^{d-1} h_{n}^{\prime \prime}(1)=\frac{(d-2)^{4} \pi^{2-d} \Gamma\left(\frac{d}{2}-1\right)^{2}}{16(d-1)^{3}} n_{s} \neq 0 .
$$

Thus problems only appear for scalars. This equation shows that the relation (4.39) and the holographic formulas (4.36), (4.37) cannot both be satisfied at the same time by free scalars.

\footnotetext{
${ }^{8}$ This is indeed the case at least in three dimensions for free fermions.
} 
That the relation (4.38) is not compatible with the holographic results can also be seen from the consideration of the positivity constraints $[19,46]$ for CFTs in general dimensions:

$$
\begin{array}{lr}
\text { Scalar Constraint : } & 1+\frac{d-3}{d-1} t_{2}+\frac{d^{2}-d-4}{d^{2}-1} t_{4} \geq 0, \\
\text { Vector Constraint : } & 1+\frac{d-3}{2(d-1)} t_{2}-\frac{2}{d^{2}-1} t_{4} \geq 0, \\
\text { Tensor Constraint : } & 1-\frac{1}{d-1} t_{2}-\frac{2}{d^{2}-1} t_{4} \geq 0 .
\end{array}
$$

These constraints are consequences of the requirement of the positivity of the energy fluxes. Now it is easy to compute from (4.36), (4.37) that

$$
\begin{aligned}
C_{D}^{\prime \prime}(1) & -d \Gamma\left(\frac{d+1}{2}\right)\left(\frac{2}{\sqrt{\pi}}\right)^{d-1} h_{n}^{\prime \prime}(1) \\
\quad= & C_{T} \frac{2 \pi^{2}(d-2)}{(d-1)^{2} d(d+1)}\left(1+\frac{d-3}{d-1} t_{2}+\frac{d^{2}-d-4}{d^{2}-1} t_{4}\right) \geq 0,
\end{aligned}
$$

where in the last step we have used the unitarity constraint $C_{T} \geq 0$ and the scalar constraint (4.44). This shows that, unless $d=2$ or if the scalar constraint is saturated, ${ }^{9}$ the relation (4.39) and our holographic results (4.36), (4.37) cannot both be satisfied at the same time.

All in all, it is therefore interesting to look for a different relation between $C_{D}^{\prime \prime}(1)$ and $h_{n}^{\prime \prime}(1)$ like those of (1.12) for the $4 \mathrm{~d}$ case and (1.21) for the $3 \mathrm{~d}$ case, that would hold for all free theories as well as strongly coupled dual theories. To do so, we need the information of $h_{n}^{\prime \prime}(1)$ and $C_{D}^{\prime \prime}(1)$ of free scalars. $h_{n}^{\prime \prime}(1)$ of free scalars is discussed in $[37,43,45]$ in general dimensions. However, so far we do not know $C_{D}^{\prime \prime}(1)$ in dimensions higher than four $(d>4)$. On the other hand, if we assume (4.39) holds for free scalars in general dimensions as has been suggested in [12], then we obtain

$$
\begin{aligned}
C_{D}^{\prime \prime}(1) & -2(d-1) \Gamma\left(\frac{d+1}{2}\right)\left(\frac{2}{\sqrt{\pi}}\right)^{d-1} h_{n}^{\prime \prime}(1) \\
& =\frac{4 \pi^{2}(d-2)}{d(d+1)} C_{T}\left[1+\frac{d-3}{d(d-1)} t_{2}+\frac{4\left(d^{2}-2 d-2\right)}{(d-1) d(d+1)(d+2)} t_{4}\right],
\end{aligned}
$$

which is such a 'universal law' obeyed by free scalars, free fermions, free conformal tensor fields and CFTs with holographic dual. Please refer to the appendix for the derivation of eq. (4.48). As a quick check, eq. (4.48) reproduces (1.12) and (1.21) for $4 \mathrm{~d}$ and $3 \mathrm{~d}$ CFTs, respectively. It is interesting to find out if the 'universal law' (4.48) is indeed valid for general CFTs. We leave this interesting problem and related questions for future work.

In summary, our holographic results (4.36), (4.37) are obeyed by free fermions and conformal tensors but are violated by free scalars. According to [12], it seems that the free CFTs satisfy (4.39). However, as we have proven above, this relation does not agree with

\footnotetext{
${ }^{9}$ The relation between (4.39) and lower bound of unitarity constraint (which is equivalent to the scalar constraint ) is observed for Gauss-Bonnet gravity for $d=4,5,6$ in [10]. Here we find this is a universal property for general higher curvature gravity in general dimensions.
} 
eqs. (4.36), (4.37). So neither the relation (4.39) nor the holographic relations (4.36), (4.37)) can be universally true for all CFTs. Instead, we find that the suitably combined relation (4.48) is satisfied by free CFTs (including scalars) as well as by CFT with holographic duals, and stands a chance to be a universal relation satisfied by all CFTs.

\section{Conclusions}

In this paper, we have investigated the universal terms of holographic Rényi entropy for $4 \mathrm{~d}$ CFTs. Universal relations between the coefficients $f_{a}^{\prime \prime}(1), f_{b}^{\prime}(1), f_{c}^{\prime}(1)$ in the logarithmic terms of Rényi entropy and the parameters $c, t_{2}, t_{4}$ of stress tensor two-point and threepoint functions are found. Interestingly, these relations are also obeyed by weakly coupled CFTs such as free fermions and vectors but are violated by scalars. Similar to the case of $f_{a}^{\prime \prime}(1)[20]$, one expects that the discrepancy for scalars comes from the boundary contributions to the modular Hamiltonian. Remarkably, We have found that there is a combination of our holographic relations which is satisfied by all the free CFTs including scalars. We conjecture that this combined relation (1.14) is universal for general CFTs in four dimensional spacetime. For general spacetime dimensions, we obtain the holographic dual of $h_{n}$ and $C_{D}$ for general higher curvature gravity. Our holographic results together with the positivity of energy flux imply $C_{D}^{\prime \prime}(1) \geq d \Gamma\left(\frac{d+1}{2}\right)\left(\frac{2}{\sqrt{\pi}}\right)^{d-1} h_{n}^{\prime \prime}(1)$. And the equality is satisfied by free fermions and the conformal tensor fields if they obey the holographic universal

laws. We also find there are similar holographic universal laws of $h_{n}^{\prime \prime}(1)$ and $C_{D}^{\prime \prime}(1)$. By assuming (4.39) for free CFTs, we find that for general dimensions, the relation (4.48) is obeyed by all the free CFTs as well as by CFTs with holographic duals. It is interesting to test these 'universal laws' by studying more general CFTs. We leave a careful study of this problem to future work.

\section{Acknowledgments}

R. X. Miao thank Yau Mathematical Sciences Center for hospitality during the early stages of this work. In particular, R. X. Miao wish to thank Prof. W. Song, Q. Wen and J. F. $\mathrm{X}$ for helpful discussions and kind help during the stay at YMSC. This work is supported in part by the National Center of Theoretical Science (NCTS) and the grant MOST 1052811-M-007-021 of the Ministry of Science and Technology of Taiwan.

\section{A Equivalence between two stress tensors}

In the analysis in the main text, we have considered in section 2 the Brown-York boundary stress tensor eq. (2.48) in section 2, and in section 4 the holographic stress tensor eq. (4.4). As we have mentioned in section 4 , they are actually equivalent up to a rescaling and some functions of $g_{(0) i j}[26]$ that are irrelevant:

$$
\left\langle T_{i j}\right\rangle=\lim _{z \rightarrow 0} \frac{1}{z^{d-2}} T_{\partial M i j} .
$$


Here the l.h.s. is the holographic stress tensor and the r.h.s. is the Brown-York boundary stress tensor. In this appendix, we shall prove that, by applying the stress tensor eq. (4.4) instead of eq. (2.48) in the approach of section 2, we obtain the same results for $f_{b}(n)$ and $f_{c}(n)$. This is can be regarded as a double check of our results.

The key point in section 2 is that the change in the partition function is govern by the stress tensor one-point function

$$
\delta \log Z_{n}=\frac{1}{2} \int_{\partial M} d x^{4} \sqrt{\gamma} T_{\partial M}{ }^{i j} \delta \gamma_{i j}
$$

From eq. (A.1) and the asymptotic AdS metric in the FG gauge eq. (4.7), one can rewrite it in terms of $\left\langle T_{i j}\right\rangle$ and $\delta g_{(0) i j}$ as

$$
\delta \log Z_{n}=\frac{1}{2} \int d x^{4} \sqrt{g_{(0)}}\left\langle T^{i j}\right\rangle \delta g_{(0) i j}
$$

The boundary metric $g_{(0) i j}$ is given by $(2.18)$ of $[10]$

$$
d s^{2}=d \tau^{2}+\frac{1}{\rho^{2}}\left(d \rho^{2}+\left[\delta_{\hat{i} \hat{j}}+2 \bar{K}_{\hat{i} \hat{j}}^{a} x_{a}+Q_{\hat{i} \hat{j}}^{a b} x_{a} x_{b}\right] d y^{\hat{i}} d y^{\hat{j}}\right)+O\left(K^{2}\right) .
$$

Actually, we can ignore the $Q$ terms above, since it is of order $O\left(K^{2}\right)$. For simplicity, we focus on the case of traceless extrinsic curvature $K_{\hat{i}}^{a \hat{i}}=0$ as in section2. Using eqs. (4.4), (4.11), we can derive the stress tensor in $\hat{i} \hat{j}$ components for 4d CFTs as

$$
\left\langle T_{\hat{i} \hat{j}}\right\rangle=\frac{4}{f_{4}} C_{T} h_{\hat{i} \hat{j}}^{(4)}=\frac{4}{f_{4}} C_{T} \frac{1}{\rho^{2}}\left[\left(\frac{1}{4} M_{e}+g_{0}\right) \delta_{\hat{i} \hat{j}}+\left(\frac{1}{2} M_{e}+2 \beta_{n}+k_{0}\right) \bar{K}_{\hat{i} \hat{j}}^{a} x_{a}\right]+O\left(K^{2}\right) .
$$

From the above two equations, we get

$$
\left\langle T^{\hat{i} \hat{j}}\right\rangle=\frac{4}{f_{4}} C_{T} h^{(4) \hat{i} \hat{j}}=\frac{4}{f_{4}} C_{T} \rho^{2}\left[\left(\frac{1}{4} M_{e}+g_{0}\right) \delta^{\hat{i} \hat{j}}+\left(-\frac{1}{2} M_{e}+2 \beta_{n}+k_{0}-4 g_{0}\right) \bar{K}^{a \hat{i} \hat{j}} x_{a}\right]+O\left(K^{2}\right) .
$$

Substituting eq. (A.6) and $\delta g_{(0) \hat{i} \hat{j}}=\frac{1}{\rho^{2}}\left(2 \delta \bar{K}_{\hat{i} \hat{j}}^{a} x_{a}+\delta Q_{\hat{i} \hat{j}}^{a b} x_{a} x_{b}\right)$ into eq. (A.3), we get

$$
\delta \log Z_{n}=\frac{1}{2} \int_{\partial M} d x^{4} \sqrt{g_{0}} T^{\hat{i} \hat{j}} \delta g_{(0) \hat{i} \hat{j}}
$$

Integrating eq. (A.7) and selecting the logarithmic divergent terms, we obtain

$$
\log Z_{n}=-\log \epsilon \int_{\Sigma} d y^{2} \frac{2 n \pi}{f_{4}} C_{T}\left[3\left(\frac{1}{4} M_{e}+g_{0}\right) C_{a b}^{a b}+\left(-\frac{1}{2} M_{e}+2 \beta_{n}+k_{0}-4 g_{0}\right) \operatorname{tr} \bar{K}^{2}\right]
$$

where we have used $C_{a b}^{a b}=\frac{1}{3} Q_{a \hat{i}}^{a \hat{i}}$ in the above derivations. Using eq. (A.8) and $M_{e}(1)=0$, we obtain the logarithmic divergent terms of Rényi entropy

$$
\begin{aligned}
S_{n} & =\frac{\log Z_{n}-n \log Z_{1}}{1-n} \\
& =\log \epsilon \frac{n}{n-1} \frac{\pi}{f_{4}} C_{T} \int_{\Sigma} d y^{2}\left[\frac{3}{2} M_{e} C_{a b}^{a b}+\left(-M_{e}+4\left(\beta_{n}-\beta_{1}\right)\right) \operatorname{tr} \bar{K}^{2}\right] \\
& =\log \epsilon \int_{\Sigma} d y^{2}\left[\frac{f_{b}(n)}{2 \pi} \operatorname{tr} \bar{K}^{2}-\frac{f_{c}(n)}{2 \pi} C^{a b}{ }_{a b}\right] .
\end{aligned}
$$


Notice that the constants $g_{0}$ and $k_{0}$ are canceled automatically in the above calcultions. Comparing eq. (A.9) and eq. (A.10) and using $C_{T} / f_{4}=\frac{c}{2 \pi^{2}}$, we finally obtain

$$
\begin{aligned}
& f_{b}(n)=\frac{n\left(4 \beta_{n}-4 \beta_{1}-M_{e}\right)}{n-1} c, \\
& f_{c}(n)=-\frac{3 n M_{e}}{2(n-1)} c .
\end{aligned}
$$

Recall that $M_{e}$ and $\beta_{n}$ are given by

$$
\begin{aligned}
M_{e} & =-\frac{2}{3}(n-1)+\frac{\left(336 c_{1}^{(2)}+192 c_{7}^{(3)}-96 c_{8}^{(3)}+35\right)}{27\left(1+8 c_{1}^{(2)}\right)}(n-1)^{2}+O(n-1)^{3} \\
\beta_{n} & =-\frac{1}{8}+\frac{n-1}{12}+\frac{\left(-600 c_{1}^{(2)}+4224 c_{7}^{(3)}-2112 c_{8}^{(3)}-67\right)}{432\left(1+8 c_{1}^{(2)}\right)}(n-1)^{2}+O(n-1)^{3} .
\end{aligned}
$$

Substituting eqs. (A.13), (A.14) and $c=\frac{\pi}{8 G_{N}}\left(1+8 c_{1}^{(2)}\right)$ into eqs. (A.11), (A.12), we reproduce the results (2.69) and (2.74) in section 2. So the stress tensor eq. (4.4) indeed yields the same results for $4 \mathrm{~d}$ CFTs as the stress tensor eq. (2.48).

Substituting $h_{n} /(n-1)=\frac{2}{3 \pi} f_{c}(n), C_{D} /(n-1)=\frac{16}{\pi^{2}} f_{b}(n)$ and $c=\pi^{4} C_{T} / 40$ [12] into eqs. (A.11), (A.12), one can also reproduce $h_{n}$ and $C_{D}$ eqs. (4.16), (4.17) for $4 \mathrm{~d}$ CFTs in section 4 .

\section{B Solutions in general higher curvature gravity}

In this appendix we provide the solutions to E.O.M of the general higher curvature gravity (2.45), which are found to be useful for the derivations of holographic $h_{n}$ and $C_{D}$ in section 4.2. For simplicity, we work in the perturbative framework of the coupling constants $c_{i}^{(n)}$. To derive $h_{n}^{\prime \prime}(1)$ and $C_{D}^{\prime \prime}(1)$ in terms of $C_{T}, t_{2}, t_{4}$, we can further set $c_{1}^{(2)}=0$ in dimensions except $d=4$. The solutions for $d=3$ and $d=4$ are given in section 4.1 and section 2.2. Below we list the key results for $d=5,6,7,8,9$ up to $O(n-1)^{2}$.

\section{5d CFTs:}

$$
\begin{aligned}
f(r)= & r^{2}-1+\frac{n-1}{2 r^{3}} \\
& -\frac{3\left(3 r^{7}\left(104 c_{7}^{(3)}-34 c_{8}^{(3)}+7\right)-8 r^{2}\left(1044 c_{7}^{(3)}-9 c_{8}^{(3)}\right)+7200 c_{7}^{(3)}\right)}{64 r^{10}}(n-1)^{2}+O(n-1)^{3}, \\
F(r)= & 1-\frac{45\left(22 c_{7}^{(3)}+c_{8}^{(3)}\right)}{4 r^{10}}(n-1)^{2}+O(n-1)^{3}, \\
k(r)= & \frac{\sqrt{r^{2}-1}}{r}+\frac{1}{r^{5}} \frac{(n-1)\left(160-(n-1)\left(-30840 c_{7}^{(3)}+15990 c_{8}^{(3)}+307\right)\right)}{3200}+O\left(\frac{1}{r^{d+1}}\right) .
\end{aligned}
$$




\section{6d CFTs:}

$$
\begin{aligned}
f(r)= & r^{2}-1+\frac{2(n-1)}{5 r^{4}} \\
& -\frac{3\left(r^{8}\left(992 c_{7}^{(3)}-232 c_{8}^{(3)}+33\right)-80 r^{2}\left(328 c_{7}^{(3)}-2 c_{8}^{(3)}\right)+23040 c_{7}^{(3)}\right)}{125 r^{12}}(n-1)^{2}+O(n-1)^{3}, \\
F(r)= & 1-\frac{72\left(132 c_{7}^{(3)}+5 c_{8}^{(3)}\right)}{25 r^{12}}(n-1)^{2}+O(n-1)^{3}, \\
k(r)= & \frac{\sqrt{r^{2}-1}}{r}+\frac{1}{r^{6}} \frac{(n-1)\left(75-2(n-1)\left(-10548 c_{7}^{(3)}+5733 c_{8}^{(3)}+73\right)\right)}{2250}+O\left(\frac{1}{r^{d+1}}\right) .
\end{aligned}
$$

\section{7d CFTs:}

$$
\begin{aligned}
f(r)= & r^{2}-1+\frac{n-1}{3 r^{5}} \\
& +\frac{\left(r^{9}\left(-7320 c_{7}^{(3)}+1320 c_{8}^{(3)}-143\right)+900 r^{2}\left(220 c_{7}^{(3)}-c_{8}^{(3)}\right)-176400 c_{7}^{(3)}\right)}{216 r^{14}}(n-1)^{2}+O(n-1)^{3}, \\
F(r)= & 1-\frac{35\left(92 c_{7}^{(3)}+3 c_{8}^{(3)}\right)}{6 r^{14}}(n-1)^{2}+O(n-1)^{3}, \\
k(r)= & \frac{\sqrt{r^{2}-1}}{r}+\frac{1}{r^{7}} \frac{(n-1)\left((n-1)\left(192696 c_{7}^{(3)}-109704 c_{8}^{(3)}-989\right)+504\right)}{21168}+O\left(\frac{1}{r^{d+1}}\right) .
\end{aligned}
$$

\section{8d CFTs:}

$$
\begin{aligned}
f(r)= & r^{2}-1+\frac{2(n-1)}{7 r^{6}} \\
& -\frac{3\left(r^{10}\left(5088 c_{7}^{(3)}-744 c_{8}^{(3)}+65\right)-504 r^{2}\left(284 c_{7}^{(3)}-c_{8}^{(3)}\right)+129024 c_{7}^{(3)}\right)}{343 r^{16}}(n-1)^{2}+O(n-1)^{3}, \\
F(r)= & 1-\frac{144\left(244 c_{7}^{(3)}+7 c_{8}^{(3)}\right)}{49 r^{16}}(n-1)^{2}+O(n-1)^{3}, \\
k(r)= & \frac{\sqrt{r^{2}-1}}{r}+\frac{1}{r^{8}} \frac{(n-1)\left((n-1)\left(194304 c_{7}^{(3)}-115392 c_{8}^{(3)}-773\right)+392\right)}{21952}+O\left(\frac{1}{r^{d+1}}\right) .
\end{aligned}
$$

\section{9d CFTs:}

$$
\begin{aligned}
f(r)= & r^{2}-1+\frac{n-1}{4 r^{7}} \\
& -\frac{3\left(r^{11}\left(9464 c_{7}^{(3)}-1162 c_{8}^{(3)}+85\right)-784 r^{2}\left(356 c_{7}^{(3)}-c_{8}^{(3)}\right)+254016 c_{7}^{(3)}\right)}{512 r^{18}}(n-1)^{2}+O(n-1)^{3}, \\
F(r)= & 1-\frac{189\left(39 c_{7}^{(3)}+c_{8}^{(3)}\right)}{8 r^{18}}(n-1)^{2}+O(n-1)^{3}, \\
k(r)= & \frac{\sqrt{r^{2}-1}}{r}+\frac{1}{r^{9}} \frac{(n-1)\left((n-1)\left(715608 c_{7}^{(3)}-441234 c_{8}^{(3)}-2279\right)+1152\right)}{82944}+O\left(\frac{1}{r^{d+1}}\right) .
\end{aligned}
$$

Using these solutions, we can derive $M_{e}$ and $\beta_{n}$ from eqs. (4.14), (4.15) as

$$
\begin{aligned}
M_{e}= & -\frac{2}{d-1}(n-1)+\frac{(2 d-3)(2 d-1)}{(d-1)^{3}}(n-1)^{2} \\
& +\frac{12(d-2)\left(d^{3}-6 d^{2}+11 d-4\right)}{(d-1)^{3}} c_{7}^{(3)}(n-1)^{2} \\
& -\frac{3(d-2)\left(3 d^{2}-9 d+4\right)}{(d-1)^{3}} c_{8}^{(3)}(n-1)^{2}+O(n-1)^{3},
\end{aligned}
$$


and

$$
\begin{aligned}
\beta_{n}= & \beta_{1}+\frac{1}{d(d-1)}(n-1)-\frac{4 d^{3}-8 d^{2}+d+2}{2 d^{2}(d-1)^{3}}(n-1)^{2} \\
& +\frac{6\left(d^{4}+2 d^{3}-21 d^{2}+36 d-16\right)}{(d-1)^{3} d} c_{7}^{(3)}(n-1)^{2} \\
& -\frac{3\left(4 d^{4}-17 d^{3}+35 d^{2}-40 d+16\right)}{2(d-1)^{3} d} c_{8}^{(3)}(n-1)^{2}+O(n-1)^{3} .
\end{aligned}
$$

Recall that we have

$$
\begin{aligned}
c_{7}^{(3)} & =\frac{2\left(d^{2}+3 d+2\right) t_{2}+(7 d+4) t_{4}}{12(d-1) d\left(d^{3}-d^{2}-10 d-8\right)}, \\
c_{8}^{(3)} & =\frac{\left(d^{2}+3 d+2\right) t_{2}+(3 d+4) t_{4}}{3 d\left(d^{4}-2 d^{3}-9 d^{2}+2 d+8\right)} .
\end{aligned}
$$

Substituting eqs. (B.6)-(B.9) into the holographic formula (4.16), (4.17), we can derive $h_{n}$ and $C_{D}$ eqs. (4.34)-(4.17) in section 4.2.

\section{Universal laws in general dimensions}

$h_{n}$ for free comformally coupled scalars in even-dimensional space-time are calculated in [45]

$$
h_{n}=\frac{(2 \pi)^{1-d}}{d-1} \sum_{j=0}^{(d-4) / 2} a_{j, l-1}^{(0)}(2 j-d+1) \pi^{2 j-d / 2}\left(n^{2 j-d+1}-n\right) \Gamma\left(\frac{d}{2}-j\right) \zeta(d-2 j),
$$

where $d=2 l+2$ and $a_{j, l-1}^{(0)}$ are defined by

$$
\begin{aligned}
P_{l-1}^{(0)}(t) & =\sum_{j=0}^{l-1} a_{j, l-1}^{(0)} t^{j} \\
& =\lim _{\rho \rightarrow 0}(4 \pi t)^{l}\left(\frac{-1}{2 \pi \sinh \rho} \frac{\partial}{\partial \rho}\right)^{l} \exp \left(-\frac{\rho^{2}}{4 t}\right) .
\end{aligned}
$$

The first few polynomials are given by

$$
\begin{aligned}
& P_{0}^{(0)}(t)=1 \\
& P_{1}^{(0)}(t)=1+\frac{2 t}{3} \\
& P_{2}^{(0)}(t)=1+2 t+\frac{16 t^{2}}{15}, \\
& P_{3}^{(0)}(t)=1+4 t+\frac{28 t^{2}}{5}+\frac{96 t^{3}}{35}, \\
& P_{4}^{(0)}(t)=1+\frac{20 t}{3}+\frac{52 t^{2}}{3}+\frac{1312 t^{3}}{63}+\frac{1024 t^{4}}{105}, \\
& P_{5}^{(0)}(t)=1+10 t+\frac{124 t^{2}}{3}+\frac{5560 t^{3}}{63}+\frac{30656 t^{4}}{315}+\frac{10240 t^{5}}{231}, \\
& P_{6}^{(0)}(t)=1+14 t+84 t^{2}+\frac{2488 t^{3}}{9}+\frac{4736 t^{4}}{9}+\frac{30208 t^{5}}{55}+\frac{245760 t^{6}}{1001} .
\end{aligned}
$$


From eq. (C.1), it is easy to derive $h_{n}^{\prime \prime}(1)$ as

$$
h_{n}^{\prime \prime}(1)=\frac{(2 \pi)^{1-d}}{d-1} \sum_{j=0}^{(d-4) / 2} a_{j, l-1}^{(0)}(2 j-d+1)^{2}(2 j-d) \pi^{2 j-d / 2} \Gamma\left(\frac{d}{2}-j\right) \zeta(d-2 j),
$$

Unfortunately, now we do not have a formula of $C_{D}(n)$. It seems that $C_{D}^{\prime \prime}(1)=$ $d \Gamma\left(\frac{d+1}{2}\right)\left(\frac{2}{\sqrt{\pi}}\right)^{d-1} h_{n}^{\prime \prime}(1)$ holds for free scalars in general dimensions [12]. This is at least the case for $d=3$ and $d=4$. With this assumption, now we are ready to derive the 'universal law' (4.48).

We require that the 'universal laws' are obeyed by both the free scalars and the holographic CFTs. From eqs. (C.4), (4.34), (4.37) and the assumption mentioned above, we finially obtain the 'universal law' (4.48) in general dimensions. In the derivations, we have used the following useful formula

$$
\sum_{j=0}^{(d-4) / 2} a_{j, l-1}^{(0)} \frac{4 d(d+2)(d-2 j-1)^{2} \pi^{-d+2 j-\frac{1}{2}} \Gamma\left(\frac{d+3}{2}\right) \zeta(d-2 j) \Gamma\left(\frac{d}{2}-j+1\right)}{(d(3 d-2)-4) \Gamma\left(\frac{d}{2}\right)^{2}}=1 .
$$

Using eq. (C.3), we verified that this identity holds up to $d=16$.

It should be mentioned that although we focus on even dimensions in the above discussions. We have checked that the 'universal law' (4.48) produces correct result (1.21) in three dimensions. So it is expected that eq. (4.48) works well in general odd dimensions too.

Open Access. This article is distributed under the terms of the Creative Commons Attribution License (CC-BY 4.0), which permits any use, distribution and reproduction in any medium, provided the original author(s) and source are credited.

\section{References}

[1] S. Ryu and T. Takayanagi, Holographic derivation of entanglement entropy from AdS/CFT, Phys. Rev. Lett. 96 (2006) 181602 [hep-th/0603001] [INSPIRE].

[2] M. Van Raamsdonk, Building up spacetime with quantum entanglement, Gen. Rel. Grav. 42 (2010) 2323 [Int. J. Mod. Phys. D 19 (2010) 2429] [arXiv:1005. 3035] [INSPIRE].

[3] N. Lashkari, M.B. McDermott and M. Van Raamsdonk, Gravitational dynamics from entanglement 'thermodynamics', JHEP 04 (2014) 195 [arXiv: 1308.3716] [INSPIRE].

[4] T. Faulkner, M. Guica, T. Hartman, R.C. Myers and M. Van Raamsdonk, Gravitation from entanglement in holographic CFTs, JHEP 03 (2014) 051 [arXiv:1312.7856] [INSPIRE].

[5] A. Lewkowycz and J. Maldacena, Generalized gravitational entropy, JHEP 08 (2013) 090 [arXiv: 1304.4926] [INSPIRE].

[6] L.-Y. Hung, R.C. Myers, M. Smolkin and A. Yale, Holographic calculations of Rényi entropy, JHEP 12 (2011) 047 [arXiv:1110.1084] [INSPIRE].

[7] X. Dong, The gravity dual of Rényi entropy, Nature Commun. 7 (2016) 12472 [arXiv: 1601.06788] [INSPIRE].

[8] P. Bueno, R.C. Myers and W. Witczak-Krempa, Universal corner entanglement from twist operators, JHEP 09 (2015) 091 [arXiv: 1507.06997] [INSPIRE]. 
[9] X. Dong, Shape dependence of holographic Rényi entropy in conformal field theories, Phys. Rev. Lett. 116 (2016) 251602 [arXiv: 1602.08493] [INSPIRE].

[10] L. Bianchi, S. Chapman, X. Dong, D.A. Galante, M. Meineri and R.C. Myers, Shape dependence of holographic Rényi entropy in general dimensions, JHEP 11 (2016) 180 [arXiv: 1607.07418] [INSPIRE].

[11] J. Camps, Gravity duals of boundary cones, JHEP 09 (2016) 139 [arXiv:1605.08588] [INSPIRE].

[12] L. Bianchi, M. Meineri, R.C. Myers and M. Smolkin, Rényi entropy and conformal defects, JHEP 07 (2016) 076 [arXiv: 1511.06713] [INSPIRE].

[13] S. Balakrishnan, S. Dutta and T. Faulkner, Gravitational dual of the Rényi twist displacement operator, arXiv:1607.06155 [INSPIRE].

[14] S.N. Solodukhin, Entanglement entropy, conformal invariance and extrinsic geometry, Phys. Lett. B 665 (2008) 305 [arXiv:0802.3117] [INSPIRE].

[15] D.V. Fursaev, Entanglement Rényi entropies in conformal field theories and holography, JHEP 05 (2012) 080 [arXiv: 1201.1702] [INSPIRE].

[16] A. Lewkowycz and E. Perlmutter, Universality in the geometric dependence of Rényi entropy, JHEP 01 (2015) 080 [arXiv:1407.8171] [INSPIRE].

[17] J. Lee, L. McGough and B.R. Safdi, Rényi entropy and geometry, Phys. Rev. D 89 (2014) 125016 [arXiv:1403.1580] [INSPIRE].

[18] D.M. Hofman and J. Maldacena, Conformal collider physics: energy and charge correlations, JHEP 05 (2008) 012 [arXiv:0803.1467] [inSPIRE].

[19] A. Buchel, J. Escobedo, R.C. Myers, M.F. Paulos, A. Sinha and M. Smolkin, Holographic GB gravity in arbitrary dimensions, JHEP 03 (2010) 111 [arXiv:0911.4257] [INSPIRE].

[20] J. Lee, A. Lewkowycz, E. Perlmutter and B.R. Safdi, Rényi entropy, stationarity and entanglement of the conformal scalar, JHEP 03 (2015) 075 [arXiv:1407.7816] [INSPIRE].

[21] P. Bueno, R.C. Myers and W. Witczak-Krempa, Universality of corner entanglement in conformal field theories, Phys. Rev. Lett. 115 (2015) 021602 [arXiv:1505.04804] [INSPIRE].

[22] M. Mezei, Entanglement entropy across a deformed sphere, Phys. Rev. D 91 (2015) 045038 [arXiv: 1411.7011] [INSPIRE].

[23] T. Faulkner, R.G. Leigh and O. Parrikar, Shape dependence of entanglement entropy in conformal field theories, JHEP 04 (2016) 088 [arXiv:1511.05179] [INSPIRE].

[24] R.-X. Miao, A note on holographic Weyl anomaly and entanglement entropy, Class. Quant. Grav. 31 (2014) 065009 [arXiv:1309.0211] [INSPIRE].

[25] V. Balasubramanian and P. Kraus, A stress tensor for anti-de Sitter gravity, Commun. Math. Phys. 208 (1999) 413 [hep-th/9902121] [INSPIRE].

[26] S. de Haro, S.N. Solodukhin and K. Skenderis, Holographic reconstruction of space-time and renormalization in the AdS/CFT correspondence, Commun. Math. Phys. 217 (2001) 595 [hep-th/0002230] [INSPIRE].

[27] Y. Brihaye and E. Radu, Black objects in the Einstein-Gauss-Bonnet theory with negative cosmological constant and the boundary counterterm method, JHEP 09 (2008) 006 [arXiv:0806.1396] [INSPIRE].

[28] C. Imbimbo, A. Schwimmer, S. Theisen and S. Yankielowicz, Diffeomorphisms and holographic anomalies, Class. Quant. Grav. 17 (2000) 1129 [hep-th/9910267] [INSPIRE]. 
[29] A. Schwimmer and S. Theisen, Entanglement entropy, trace anomalies and holography, Nucl. Phys. B 801 (2008) 1 [arXiv:0802.1017] [InSPIRE].

[30] R.-X. Miao, Universal terms of entanglement entropy for 6d CFTs, JHEP 10 (2015) 049 [arXiv: 1503.05538] [INSPIRE].

[31] R.-X. Miao, A holographic proof of the universality of corner entanglement for CFTs, JHEP 10 (2015) 038 [arXiv: 1507.06283] [INSPIRE].

[32] K. Sen and A. Sinha, Holographic stress tensor at finite coupling, JHEP 07 (2014) 098 [arXiv: 1405.7862] [INSPIRE].

[33] A. Naseh, Scale vs conformal invariance from entanglement entropy, arXiv:1607.07899 [INSPIRE].

[34] S. Cremonini, J.T. Liu and P. Szepietowski, Higher derivative corrections to R-charged black holes: boundary counterterms and the mass-charge relation, JHEP 03 (2010) 042 [arXiv:0910.5159] [INSPIRE].

[35] N. Deruelle, M. Sasaki, Y. Sendouda and D. Yamauchi, Hamiltonian formulation of f(Riemann) theories of gravity, Prog. Theor. Phys. 123 (2010) 169 [arXiv:0908.0679] [INSPIRE].

[36] I.R. Klebanov, S.S. Pufu, S. Sachdev and B.R. Safdi, Rényi entropies for free field theories, JHEP 04 (2012) 074 [arXiv:1111.6290] [INSPIRE].

[37] H. Casini and M. Huerta, Entanglement entropy for the n-sphere, Phys. Lett. B 694 (2011) 167 [arXiv:1007.1813] [inSPIRE].

[38] D.V. Fursaev and G. Miele, Finite temperature scalar field theory in static de Sitter space, Phys. Rev. D 49 (1994) 987 [hep-th/9302078] [INSPIRE].

[39] L. De Nardo, D.V. Fursaev and G. Miele, Heat kernel coefficients and spectra of the vector Laplacians on spherical domains with conical singularities, Class. Quant. Grav. 14 (1997) 1059 [hep-th/9610011] [INSPIRE].

[40] H. Osborn and A.C. Petkou, Implications of conformal invariance in field theories for general dimensions, Annals Phys. 231 (1994) 311 [hep-th/9307010] [INSPIRE].

[41] J. Erdmenger and H. Osborn, Conserved currents and the energy momentum tensor in conformally invariant theories for general dimensions, Nucl. Phys. B 483 (1997) 431 [hep-th/9605009] [INSPIRE].

[42] M. Billò, V. Gonçalves, E. Lauria and M. Meineri, Defects in conformal field theory, JHEP 04 (2016) 091 [arXiv: 1601. 02883] [INSPIRE].

[43] J.S. Dowker, Conformal weights of charged Rényi entropy twist operators for free scalar fields, arXiv: 1508.02949 [INSPIRE].

[44] J.S. Dowker, Conformal weights of charged Rényi entropy twist operators for free Dirac fields in arbitrary dimensions, arXiv:1510.08378 [INSPIRE].

[45] L.-Y. Hung, R.C. Myers and M. Smolkin, Twist operators in higher dimensions, JHEP 10 (2014) 178 [arXiv:1407.6429] [INSPIRE].

[46] X.O. Camanho and J.D. Edelstein, Causality constraints in AdS/CFT from conformal collider physics and Gauss-Bonnet gravity, JHEP 04 (2010) 007 [arXiv:0911.3160] [INSPIRE]. 